\title{
Aplicaciones de sintaxis espacial en Arqueología: una revisión de algunas tendencias actuales
}

\section{Space Syntax Applications In Archaeology: A Review Of Some Current Trends}

\author{
Jesús Bermejo Tirado \\ History Department, York University \\ jesusbermejotirado@gmail.com
}

\begin{abstract}
RESUMEN
Este trabajo realiza un recorrido por algunas de las principales tendencias teóricas y metodológicas sobre la aplicación de técnicas de análisis sintáctico-espacial para el estudio de edificios y asentamientos arqueológicos. Hemos divido nuestra exposición en una serie de claves temáticas que sirven para agrupar los trabajos y autores comentados. Nuestro análisis nos permite obtener, por primera vez, una síntesis comprensiva sobre la orientación actual de este tipo de aplicaciones en el ámbito de la Arqueología actual. Para concluir nuestro trabajo planteamos una serie de propuestas de futuro que creemos que contribuirán a asentar este tipo de aplicaciones arqueológicas como un elemento destacado en los debates en torno a la ciudad como uno de los problemas fundamentales del ser humano en el siglo XXI.
\end{abstract}

Palabras clave: Arqueología de la arquitectura; Space Syntax; Urbanismo antiguo; Ciudades arqueológicas; Configuración arquitectónica.

\section{ABSTRACT}

This paper reviews some current trends on the application of Space Syntax methodologies for the analysis of Archaeological built environments. It has been divided into different thematic points to approach the explanation of related publications and authors in a more comprehensible way. The study of all these works allows establishing the first synthesis on this issue in the current archaeological bibliography. Finally, I propose a serie of application trends that must be faced in the general framework of current debates on the City as one of the main problems in the XXI century.

Keywords: Archaeology of Architecture; Space Syntax; Ancient Urbanism; Archaeological cities; Architectonical configuration.

Recibido: 19-05-2015. Aceptado: 03-08-2015.

\section{Cómo citar este artículo / Citation}

Bermejo Tirado, J. 2015: "Aplicaciones de sintaxis espacial en Arqueología: una revisión de algunas tendencias actuales", Arqueología de la Arquitectura, 12: e031. http://dx.doi.org/10.3989/arq.arqt.2015.122

\section{Copyright}

(c) 2015 CSIC. Este es un artículo de acceso abierto distribuido bajo los términos de la licencia Creative Commons Attribution-Non Commercial (by-nc) Spain 3.0 


\section{Introducción}

En las últimas décadas hemos asistido a la publicación de un número creciente de trabajos sobre la aplicación de técnicas de análisis sintáctico-espacial en entornos construidos de diversos periodos del pasado. La capacidad de este tipo de métodos para explicitar las dinámicas o conductas sociales que subyacen en la configuración espacial de los edificios, asentamientos y paisajes creados por el ser humano ha sido recientemente explotada por arqueólogos de diversa orientación metodológica y teórica.

El hecho de que este tipo de herramientas de análisis puedan ser utilizadas como base para establecer inferencias e interpretaciones sobre la vida social de individuos y comunidades de todo tipo, incluídas aquellas que no han dejado documentación textual sobre estos aspectos, ha contribuido al progresivo interés por su aplicación en diversos contextos prehistóricos. Al mismo tiempo, el uso de este tipo de herramientas en contextos históricos se está revelando como una herramienta muy eficaz a la hora de generar interpretaciones historiográficas alternativas, o abiertamente críticas, con las que matizar las narrativas tradicionales (p. e. Vaughan, Clark, Sahbaz y Haklay 2005).

Pero a pesar de que este creciente interés está empezando a cristalizar en forma de encuentro ${ }^{1}$, conferencias, tesis doctorales y publicaciones específicas (como este monográfico; también cf. Paliou, Lieberwirth y Polla 2014), todavía no contamos con ningún trabajo de síntesis que aborde la revisión sistemática de las tendencias reflejadas en los trabajos publicados hasta la fecha (Smith 2011: 176). Con esta intención, la de ofrecer una síntesis reflexiva de las obras que se han ido publicando en los últimos años, hemos creído pertinente la elaboración de este trabajo.

El alto nivel de abstracción al que debemos enfrentarnos todos los arqueólogos interesados en el uso de este tipo de herramientas de análisis ha generado un cierto ánimo pedagógico que se refleja en gran parte de las publicaciones sobre este tipo de aplicaciones. En cierto sentido, nos vemos avocados a tener que explicar, si queremos trascender las fronteras del reducido público introducido a estas cuestiones, el funcionamiento

\footnotetext{
${ }^{1}$ En este sentido cabe resaltar que el último simposio sobre sintaxis espacial (10th Space Syntax Symposium) que se celebró entre los días 13 y 17 de Julio de 2015 en Londres (UCL), contó, por primera vez en la historia de estos encuentros, con una sesión específica dedicada a las aplicaciones arqueológicas de sintaxis espacial.
}

de este tipo de herramientas de análisis. En muchos casos, esta tendencia a explicar el cómo, por otra parte fácilmente comprensible, llega incluso a eclipsar la discusión del porqué de dichos estudios. Sin embargo, al hacer esto corremos el riesgo de convertirnos en una especie de comisarios políticos o de exégetas oficiales de una ortodoxia transmitida en los textos de los "padres fundadores". Nada más lejos de la realidad actual de un campo, el de la sintaxis espacial, que en los últimos años se ha convertido en un floreciente foco de investigación (verdaderamente) interdisciplinar. A diferencia de otras tendencias metodológicas o teóricas, la sintaxis espacial está sometida a un continuo proceso centrífugo/centrípeto de reelaboración de críticas y creación de nuevas herramientas de análisis y aplicaciones en los más diversos campos de las ciencias sociales y humanas. Tanto es así que, en algunos casos, los arqueólogos nos hemos apropiado la capacidad de inventar nuevas formas de análisis que, aunque inspiradas en principios sintácticos previos, pueden considerarse como aportaciones absolutamente originales (Blanton 1994; Grahame 2000: 56-57; Fisher 2009: 444-445).

Por todos estos motivos, este trabajo, más que intentar medir el grado de comprensión de las técnicas de sintaxis espacial por parte de algunos arqueólogos, se va a concentrar en analizar la orientación final de estas aplicaciones en tanto que trabajos de Arqueología. Para conseguir de forma más efectiva este objetivo hemos agrupado todas las aplicaciones arqueológicas de técnicas sintáctico-espaciales a las que hemos tenido acceso en una serie de grupos temáticos.

\section{2. "Space Syntax es...": introducciones sintáctico-espaciales}

Como ya hemos señalado, uno de los principales problemas a los que nos enfrentamos los practicantes de este tipo de aplicaciones radica en que, muy a menudo, los resultados de nuestros trabajos deben ser presentados ante audiencias que desconocen los más elementales fundamentos de estas estrategias de análisis. Por este motivo, muchas publicaciones arqueológicas sobre la aplicación de técnicas de sintaxis espacial suelen incluir capítulos introductorios con objeto de explicar los rudimentos metodológicos de sus herramientas de análisis. En muchos casos estos trabajos se convierten en verdaderas guías sintéticas de los que podríamos considerar como trabajos seminales (Benedikt 1979; 
Hillier y Hanson 1984, 1987; Hillier 1985, 1989, 1996; Hillier, Hanson y Graham 1987; Hillier, Hanson y Peponis 1987; Hillier 1993; Hanson 1998).

Por supuesto, la lectura de estas introducciones o definiciones iniciales no pueden sustituir en modo alguno el estudio dichos trabajos seminales, así como de las publicaciones específicas sobre sintaxis espacial $\mathrm{y}$, por supuesto, algunas de las críticas clásicas a este tipo de metodologías ${ }^{2}$. Sin querer presentar una nueva y redundante explicación, creemos pertinente realizar algunos comentarios sobre este tipo de introducciones con objeto de caracterizar algunos patrones relevantes en el marco de las aplicaciones arqueológicas.

Un primer grupo de estas introducciones estaría compuesto por aquellos textos escritos por arquitectos interesados en el análisis de edificios y ciudades históricas o arqueológicas. Dentro de este grupo habríamos de situar el frecuentemente citado trabajo de Bafta (2003). Se trata de un artículo muy sintético donde podemos encontrar una completa explicación de los principales índices cuantitativos y las características básicas (sobre todo a nivel gráfico) de los análisis alpha y gamma. Otro trabajo más reciente y mucho más completo desde un punto de vista metodológico, es el publicado por Akkelies van Nes (2014) en el marco de un volumen más amplio sobre geodiseño y ciencias geoespaciales (Lee, Dias y Scholten 2014). En ambos trabajos se recogen recursos gráficos muy útiles desde un punto de vista pedagógico. Sin embargo, ambos adolecen de una cierta falta de profundidad sobre las raíces teóricas de estas formulaciones. Este hecho es especialmente relevante en el caso de las aplicaciones arqueológicas ya que, en algunos casos, se asumen principios teóricos (como la relación entre profundidad (Depth) y privacidad) asociados a ciertos principios sintáctico-espaciales de forma automática.

Otro grupo de estas introducciones es el conformado por aquellos trabajos firmados por arqueólogos. En él se incluye un conjunto de trabajos muy amplio ya que prácticamente en la totalidad de las publicaciones arqueológicas sobre este tema se incluyen pasajes introductorios de este tipo. Hace algunos años, en esta misma revista, tuvimos la oportunidad de publicar un artículo sobre esta misma cuestión (Bermejo 2009). En él tratábamos de realizar una genealogía reflexiva sobre

\footnotetext{
2 Proceedings of the Space Syntax Symposia, Journal of Space Syntax, también vid. Leach 1978; Blanton 1994; Steadman 1996; Orhum, Hillier y Hanson 1996; Osman y Sulivan 1994, 1995; Turner, Doxa, O’Sullivan y Penn 2001; Penn 2003; Al Sayed, Turner, Hillier, Iida y Penn 2014.
}

las raíces teóricas de la sintaxis espacial, además de introducir las principales herramientas de representación gráfica y los índices analíticos consignados en los trabajos seminales anteriormente consignados. Revisando este artículo, encuentro que dicho trabajo contiene una carencia fundamental. Hubiera sido conveniente introducir un punto relativo a los principios matemáticos, sobre todo los relativos a la teoría del grafo y la geometría oculta, de la sintaxis espacial. Esto hubiera permitido contextualizar con mayor precisión las génesis de muchas herramientas de análisis sintáctico-espacial. Afortunadamente, disponemos de otro magnífico capítulo introductorio, enmarcado en un libro publicado por Shapiro (2005: 36-58) en el que se aborda la discusión de los fundamentos geométricos de la sintaxis espacial de un modo accesible para cualquier lector no introducido en la materia. Especialmente interesante resulta la genealogía léxica de conceptos como los análisis alpha y gamma, rastreando la influencia de trabajos fundamentales, no explicitados previamente, como el volumen de Taaffe y Gauthier (1973) sobre la relación entre la geometría y la geografía humana.

Además de estos dos grupos, queremos introducir un comentario sobre la reciente publicación de un texto de Hillier (2014) sobre la necesidad de introducir modelos teóricos en los análisis sintáctico-espaciales aplicados en contextos arqueológicos. A pesar de la comprensible falta de familiaridad con los detalles técnicos de la investigación actual de determinadas realidades arqueológicas que aparecen vinculadas a su propia agenda investigadora ${ }^{3}$, lo cierto es que este trabajo contiene una explicación comprehensiva de algunas de las más importantes concepciones teóricas de la sintaxis espacial como método de análisis arquitectónico. Su lectura resulta especialmente esclarecedora sobre la concepción geométrica del espacio social, lo que sirve de fundamento a gran parte de sus paradigmas interpretativos para el fenómeno urbano.

\section{Genotipos sociales}

En este apartado se agrupan todos aquellos trabajos que utilizan técnicas sintáctico-espaciales con objeto

\footnotetext{
${ }^{3}$ Cuestión que debería llevarnos a una reflexión mucho más profunda, para la que no tenemos espacio en estas líneas, sobre las causas de que la investigación arqueológica más reciente sea sistemáticamente ignorada en al marco de algunos de los más importantes debates en ciencias sociales como los que se producen en torno al urbanismo actual.
} 
de registrar la materialización de determinadas dinámicas o conductas sociales a través de análisis de la configuración espacial de los entornos construidos. En un trabajo clásico, Hillier, Hanson y Graham (1987: 363), propusieron el concepto de genotipo social, es decir, el conjunto de características sintáctico-espaciales que presentan determinados edificios en función de su contexto o función social. Sobre la base de esta definición, cada vez más arqueólogos están desarrollando diferentes estrategias de análisis con objeto de identificar el genotipo social de diversas tipologías edilicias del pasado.

Se trata, con diferencia, del grupo más numeroso de estudios en el marco de este tipo de aplicaciones. Dentro de él englobamos, por un lado, aquellas aplicaciones destinadas al establecimiento de genotipos sociales en el marco de contextos culturales prehistóricos o ágrafos (Van Dyke 1999; Dawson 2002; Stockett 2005; Vaquer y Nielsen 2011; Grau 2013; Nevadomsky, Lawson y Hazlett 2014), y por el otro, aquellos estudios de edificios históricos pertenecientes a contextos en los que es posible deducir modelos de conducta social a través de fuentes escritas (Fairclough 1992; Mol 2012). En este segundo grupo, como veremos en detalle más adelante, la aplicación de estrategias de análisis sintáctico espacial ha demostrado ser muy efectiva a la hora de registrar genotipos sociales divergentes a los paradigmas canónicos, que normalmente eclipsan otras formas de conducta. Asimismo, en otros casos concretos, la aplicación de este tipo de técnicas nos ha permitido completar nuestra percepción de aspectos poco conocidos, en el marco de normativas sociales bien documentadas (Chatford Clark 2007).

El análisis de conjuntos palaciegos o grandes residencias en de las diversas culturas del Bronce egeo puede ser utilizado como paradigma del primer subgrupo planteado. En este sentido, los trabajos de Fisher $(2009,2014)$ sobre algunos edificios del Bronce Final chipriota, como el complejo Ashlar de Enkomi (fig. 1), nos han permitido conocer que estos edificios también estaban destinados a facilitar el desarrollo de distintos tipos de interacción social dentro de estas comunidades. Sus trabajos nos muestran de qué modo el diseño de estos edificios incluía diversas características espaciales (como la conexión de espacios singulares o la construcción de salas para la celebración de distintos tipos de comitivas y reuniones) destinadas a articular interacciones sociales entre diversos grupos de la comunidad. El control espacial de estas relaciones sociales era clave para la hegemonía de las elites que construyeron este tipo de edificios.

En esta misma línea, resulta muy significativo el interés suscitado por el estudio sintáctico-espacial de los palacios minoicos. El análisis de estos complejos ha sido abordado por Letesson $(2009,2013,2014 a, 2014 b)$ en varios trabajos recientes en los que se reinterpreta la configuración espacial de varios palacios durante la fase Neopalacial cretense (1600-1425 a. C.). Los datos ofrecidos a partir del empleo de diversas técnicas de representación gráfica, como los gráficos de accesibilidad o los gráficos e interacción visual (VGA) (fig. 2), le han permitido reconocer un genotipo configuracional en la arquitectura neopalacial orientada a la creación de nodos de interacción que sirvieron como espacios de relación entre los llamados palacios y las casas circundantes a estos complejos. Según este autor, frente a la arquitectura de los periodos anteriores (pre y proto-palacial), caracterizada por el surgimiento del modelo palaciego de forma segregada a su entorno, la arquitectura de los grandes conjuntos neopalaciales debería entenderse como entornos construidos en los que los palacios se encuentran integrados con el resto de áreas domésticas dentro de estos asentamientos en función de una dinámica de configuración. Desde este punto de vista el palacio no era tanto un núcleo de proyección social de las elites minoicas como la materialización de una nueva forma de articular la comunidad. Un reciente trabajo de este mismo autor (Letesson 2014b) sirve para remarcar el papel que los hogares y kernoi, situados en dichas áreas de interacción, tenían dentro de estas dinámicas configuracionales neopalaciales.

También relevantes, en el marco de esta revisión sintáctico-espacial de la arquitectura palaciega del Bronce egeo, son los trabajos de Hacigüezeller y Thaler (Thaler 2005; Hacigüzeller 2008; Hacigüzeller y Thaler 2014). Estos trabajos parten de la premisa de que la aplicación de estrategias analíticas tipo Space Syntax, del mismo modo que en el caso de los SIG, no tiene por qué estar vinculada a ninguna de las orientaciones teóricas previamente establecidas (Hillier 2014). En su estudio comparativo de la arquitectura palacial del Bronce Final en Malia (Creta) y Pilos (Mesenia) (Hacigüzeller y Thaler 2014) se infieren dos formas diferentes de articular la circulación dentro de los palacios. Mientras que en Malia los índices de integración son altos en todas las fases (Hacigüzeller y Thaler 2014: 227), a medida que el edificio va sufriendo reformas durante los últimos periodo del Bronce Final, se observa cierta tendencia a la compartimentación funcional. 


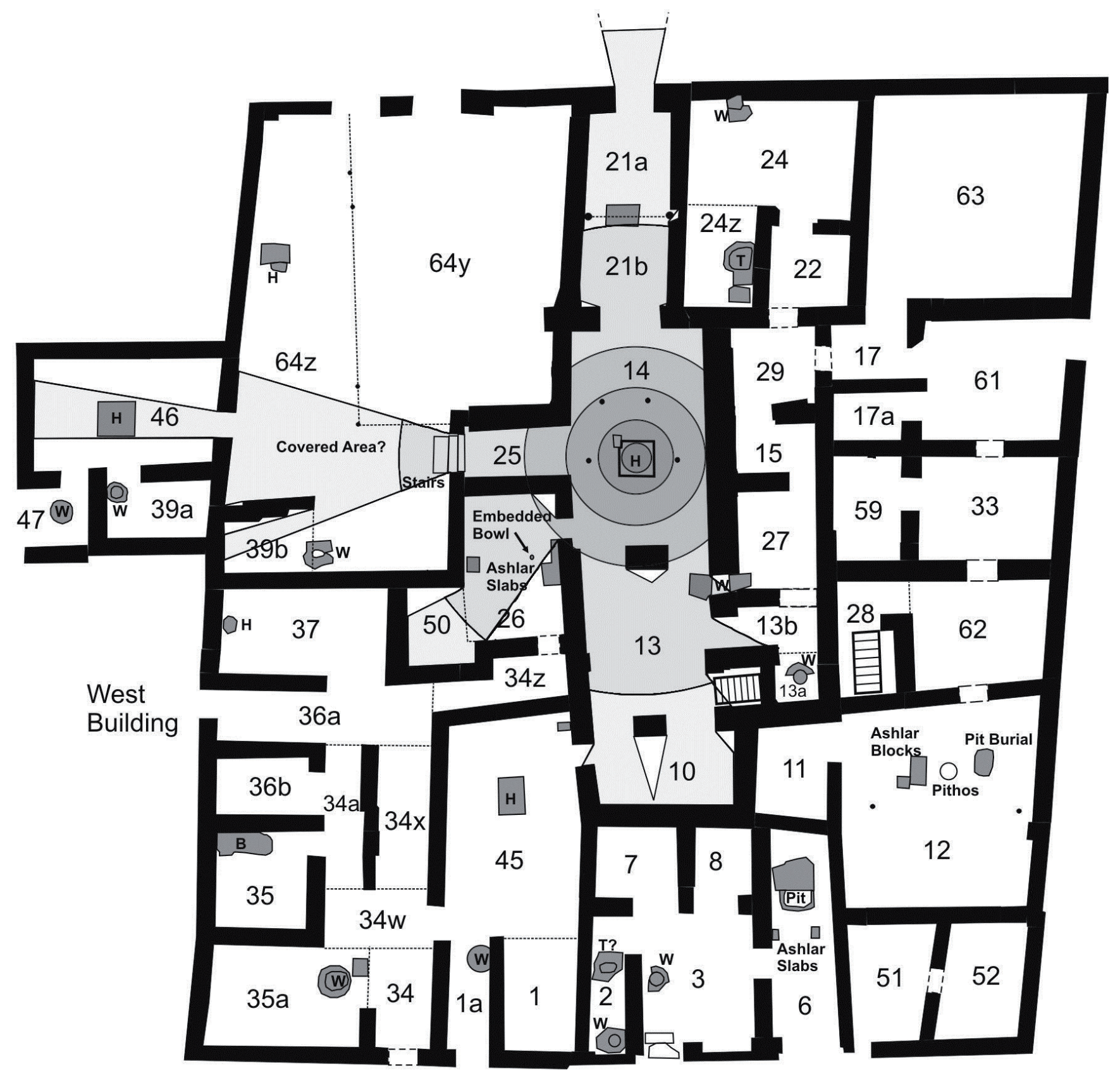

\begin{tabular}{|c|}
\hline $\begin{array}{l}\text { Feature (W=well; } \mathrm{H}=\text { hearth; } \\
\text { T=toilet; } \mathrm{B}=\text { bench) }\end{array}$ \\
\hline Conjectured Doorway \\
\hline $\begin{array}{l}\text { ए⿴囗⿱一一) Stairway } \\
\text { Boundary of Convex Space }\end{array}$ \\
\hline
\end{tabular}

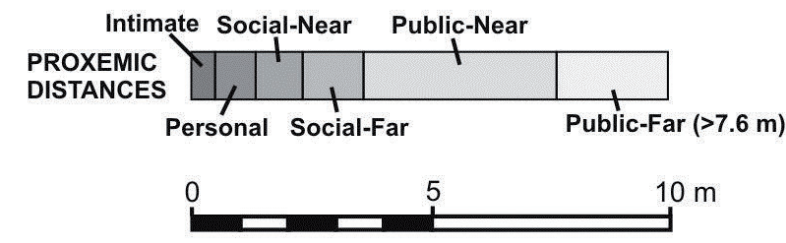

Figura 1. Plano esquemático de la Fase del Bronce Final IIIA del Complejo Ashlar, Enkomi (Chipre) que muestra un gráfico de distancias proxémicas generadas a partir de una isovista cuyo punto generador es el altar central (H) del edificio. A partir de Fisher 2009 : Fig. 8. 

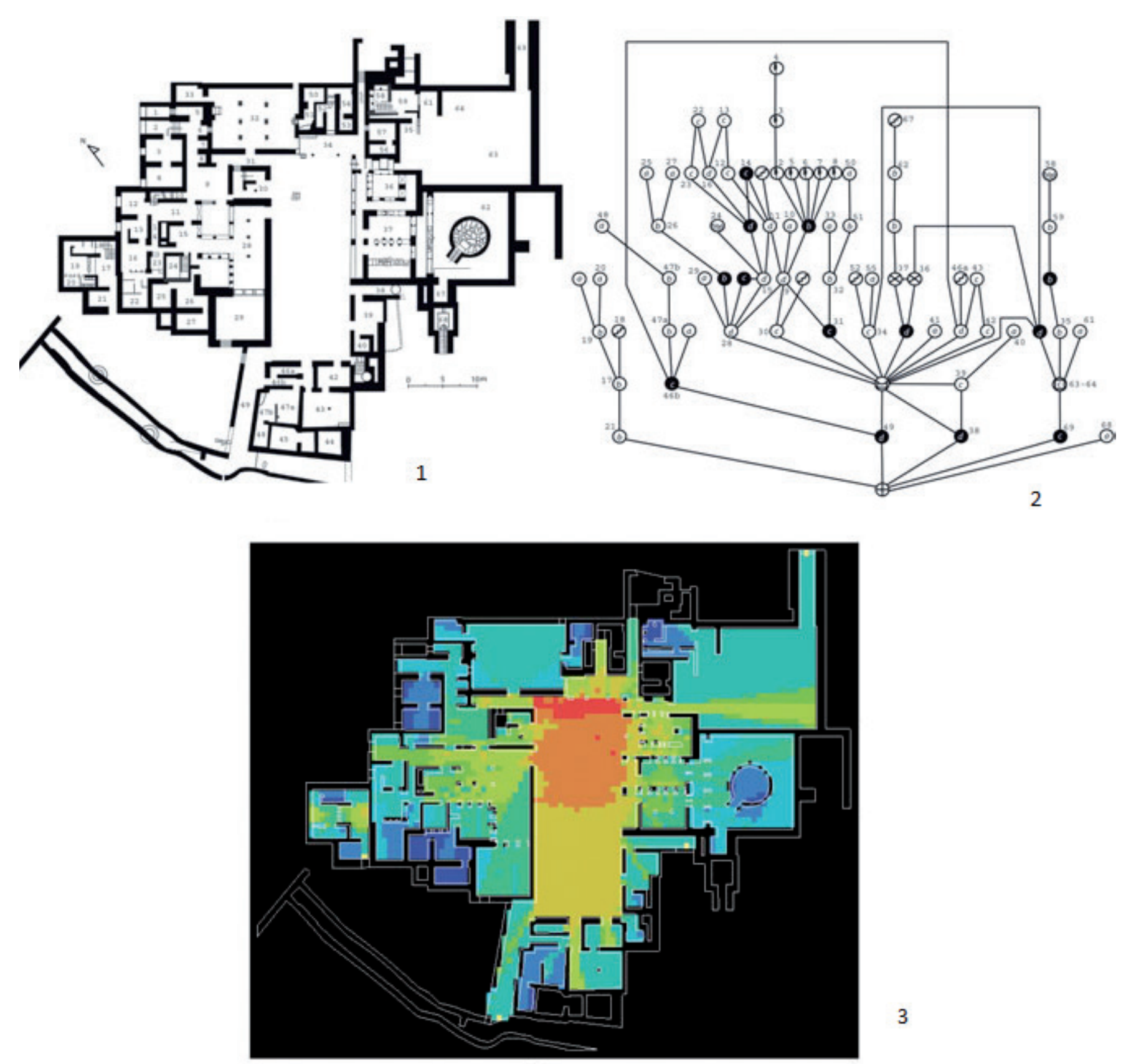

3

Figura 2. 1: Planimetría esquemática de la Fase II del Palacio minoico de Zakros. 2: Gráfico de accesibilidad. 3: Gráfico de integración visual. A partir de Letesson 2014: Fig. 3.

En el caso de la Pilos micénica, a lo largo de todas las etapas se observa cierta tendencia a la diversidad de recorridos de las principales rutas de circulación y acceso a los distintos ámbitos del complejo (Hacigüzeller y Thaler 2014: 245-247). Esto se interpreta como una tendencia a la diferenciación de los diversos ambientes en relación a su actividad funcional o económica dentro del complejo. La comparación conjunta de ambos casos desde una perspectiva diacrónica sugiere un aumento progresivo de los índices de integración en las sucesivas ampliaciones de ambos complejos edilicios a lo largo de las últimas fases del Bronce Final minoico.

Como hemos señalado al principio de este punto, la aplicación de técnicas de análisis sintáctico-espacial también se ha utilizado para la caracterización de genotipos sociales en el marco de culturas históricas. En algunos casos, dichos trabajos han servido para poner de manifiesto realidades divergentes a modelos canónicos estudiados a través de las fuentes escritas. En este punto nos gustaría destacar una serie de estudios que se 
han encargado de analizar la arquitectura doméstica de época clásica (griega y romana). Un artículo de R. Westgate (2007) ha servido para poner de manifiesto cómo los modelos canónicos de la arquitectura residencial urbana de las polis clásico-helenísticas de centros como Atenas, Delos y Olinto (Pesando 1989) coexistieron con otras formas de concebir la casa griega. Su trabajo utiliza como contrapunto la arquitectura doméstica de varias ciudades clásicohelenísticas en la isla de Creta como Lato, Trypetos o Praisos. Pero, más allá de constatar esta variabilidad, lo verdaderamente destacable del trabajo de Westgate es que logra articular una muy convincente hipótesis interpretativa para interpretar estas diferencias en clave sociológica. Las casas del modelo canónico están diseñadas para albergar unidades domésticas en las que la concepción de ciudadanía está fuertemente relacionada con la reproducción sexual de los cónyuges, pertenecientes a un estatus cívico determinado: el de ciudadanos, en el exclusivo marco de un matrimonio legalmente sancionado por la autoridad política. En el caso cretense, según Westgate, la concepción de la ciudadanía estaría relacionada con la pertenencia efectiva a la comunidad, no tanto regulada en función de unas relaciones de parentesco sancionadas por la ciudad-estado como por la participación de las familias en las tareas y obligaciones comunitarias, como se atestigua en diversas fuentes escritas (Westagate 2007: 448-453).
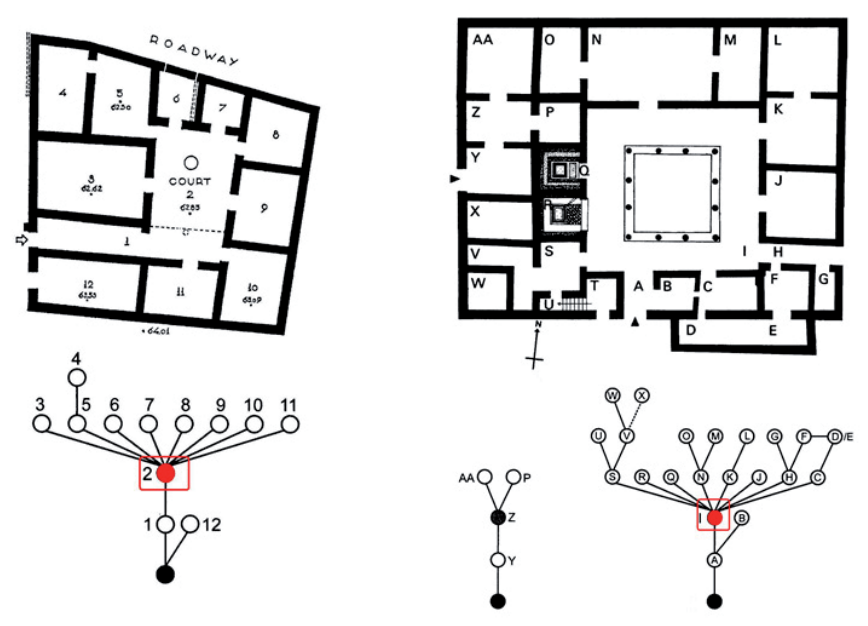

Figura 3. Planimetría y gráficos de accesibilidad de la Casa D del Agora de Atenas (Siglo V a. C.) y de las Casa de los Pendones y Casa de los Comediantes (Siglo II a. C.) de Delos con gráficos de accesibilidad en los que se puede observar la presencia de espacios controladores. A partir de Westgate 2007: Fig. 1.
La vinculación de ciudadanía y control sexual de la reproducción en el matrimonio impuso la necesidad de modelos domésticos con espacios controladores (fig. 3) como recurso panóptico para regular la vida social en el marco de los oikoi de los ciudadanos de una parte de las polis ático-délicas. No debemos olvidar que, a diferencia de lo que ocurre con las actuales familias nucleares occidentales, en el seno de las unidades domésticas de la Antigüedad clásica, sobre todo en el caso de las elites, se daba una convivencia espacial efectiva de múltiples individuos (familares, esclavos, dependientes, trabajadores, invitados, etc.). Las obligaciones sociales y económicas de estas unidades domésticas provocaron la necesidad de someter a las esposas e hijas de ciudadanos (garantes de la correcta transmisión de los derechos de ciudadanía a través de una reproducción sexual regulada en el marco del matrimonio legalmente sancionado) a la posibilidad de un escrutinio continuo dentro de la propia casa. La regulación espacial de este tipo de conductas sociales (y sexuales) ayuda a explicar el hecho de que el surgimiento del modelo canónico de la casa con patio (panóptico) ocurra fundamentalmente en aquellas regiones en las que, como en Atenas, tenemos noticia del surgimiento de este tipo de estructuras políticas.

Por el contrario, en el caso cretense, o en otros contextos del área cultural griega clásica como por ejemplo la ciudad de Colofón, en la región jonia del Asia Menor (Pesando 1989: fig. 60), la presencia de formas de regulación cívica alternativa quedó materializada en el rechazo de la adopción de estas características sintácticoespaciales, así como en el mantenimiento de modelos lineales arcaicos (Westgate 2007: 445-448; Bintliff 2014) en los que no aparecen espacios controladores, para las casas de estos asentamientos, incluso para las que podrían haber pertenecido a las elites locales (fig. 4).

En el marco del periodo romano, varios trabajos de Grahame $(1997,2000)$ han servido para probar que el modelo canónico de la domus pompeyana, se debe más a una construcción historiográfica que a la presencia generalizada de un modelo tipológico unitario, incluso en la propia Pompeya. Sus estudios han servido para plantear que, el modelo vitruviano de la arquitectura doméstica romana, estaba relacionado con la política social del periodo altoimperial, destinado a regular la concepción paterfamiliar de la ciudadanía romana (Milnor 2005: 94139), del mismo modo que el régimen victoriano estaba estrechamente relacionado con una concepción moral de la vida cotidiana. 


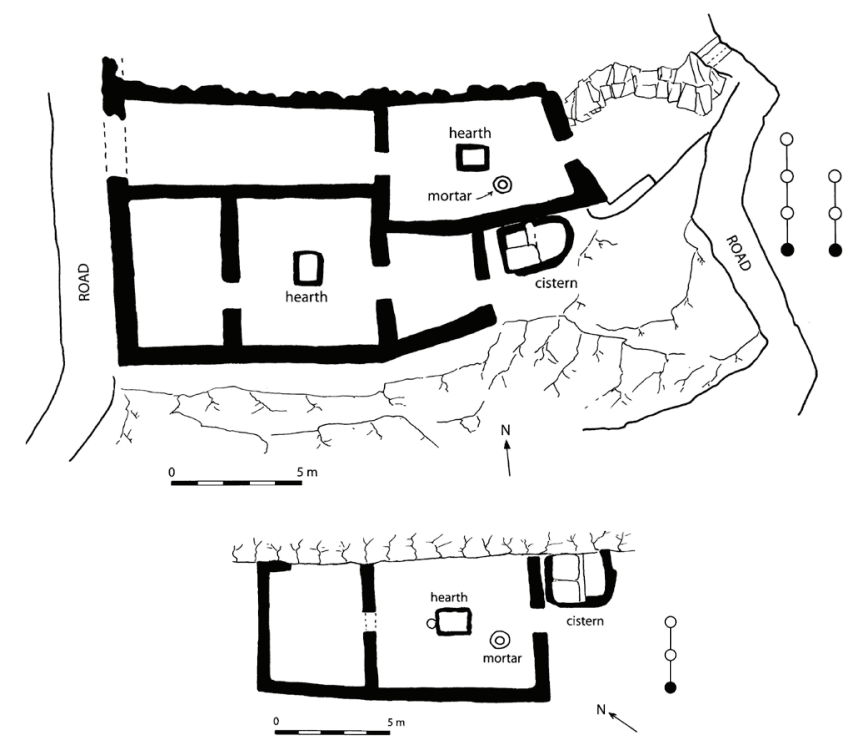

Figura 4. Arriba. Casas del Norte del Pritaneo (Lato, Creta). S. IV a. C. A partir de Westgate (2007a). Gráfico de accesibilidad. Abajo. Casa $\Delta$. Lato (Creta) S. IV a. C. A partir de Westgate (2007a). Gráfico de accesibilidad.

En todos estos casos hemos podido comprobar de qué modo el desarrollo de modelos normativos de conducta doméstica (o su rechazo) en función de diversos tipos de regímenes político-sociales se materializa a través de la configuración sintáctico-espacial de los diversos tipos de arquitectura privada desarrollados por una comunidad. Pero, más relevante aún que la capacidad de reconocer el impacto de los modelos normativos en el diseño arquitectónico, es que la aplicación de técnicas de análisis tipo Space Syntax nos permite establecer genotipos sociales específicos para cada caso de estudio. A diferencia de los modelos canónicos tradicionales, que sólo pueden establecer interpretaciones histórico-sociales a partir del parecido de nuevos casos en relación a tipologías preestablecidas, el análisis sintácticoespacial puede ser utilizado para inferir el genotipo social de un edificio concreto de forma individualizada.

También en el marco de los estudios centrados en contextos de época romana, otros autores se han encargado de analizar otras dinámicas sociales materializadas por medio de la configuración arquitectónica de entornos construidos. Dentro de este apartado quisiéramos destacar especialmente los trabajos de J. Stöger $(2011,2014)$ (véase su capítulo dentro de este mismo monográfico), que nos han permitido, entre otras cosas, conocer el impacto de la configuración de comunidades vecinales en el entorno ostiense como un factor fundamental a la hora de entender la configuración sintáctico-espacial de determinados sectores urbanos.
El análisis sintáctico-espacial de edificios de época medieval también ha sido objeto de atención por varios autores en los últimos años (Chatford Clark 2007; Cañavate 2008, 2013; Gutiérrez y Cánovas 2009; Mol 2012). Desde un punto de vista metodológico quisiéramos destacar un reciente estudio a cargo de Paliou (2014) sobre el análisis de la conectividad visual en el interior de la iglesia bizantina de San Vital de Ravenna. La aplicación de este tipo de técnicas de análisis de isovistas integradas a partir de modelos 3D (fig. 5) le ha permitido matizar el patrón de segregación por género que subyace en el genotipo social del diseño de este edificio, como escenario de la liturgia cristiana de época altomedieval. $\mathrm{Su}$ estudio ha permitido caracterizar nuevos escenarios de relación visual entre géneros y las relaciones de dominación visual de los diversos sectores del edificio. Dichas relaciones generan modos de interacción social más complejos que los que deja traslucir la estricta normativa litúrgica reflejada en los textos canónicos (Paliou 2014: 107-108) y que no pueden ser registrados a través del cotejo de las tradicionales planimetrías en 2D.

\section{Configuración urbanística en el pasado}

El estudio de la ciudad como fenómeno histórico ha sido un aspecto esencial para el desarrollo de la Space Syntax como método de análisis (Hillier y Vaugan 2007). Muchos autores se han preocupado por utilizar este tipo de estrategias de análisis para cotejar de qué modo la configuración espacial de las ciudades estaba influida por las actividades de sus habitantes y viceversa (Van Nes 2014).

En el marco de la Arqueología, la aplicación de este tipo de estrategias de análisis para el análisis de la configuración urbana se ha circunscrito fundamentalmente a casos de ciudades destruidas y abandonadas, excavadas posteriormente en extensión. Por este motivo no debe extrañarnos que la mayor parte de aplicaciones arqueológicas se hayan centrado en ciudades de la Antigüedad romana, las culturas del Oriente Próximo antiguo y en determinados contextos urbanos de la América prehispánica, aunque también contamos con algún estudio referido a contextos medievales (Craane 2007). Gran parte de estos estudios han abordado el estudio sintáctico-espacial de los modos de circulación que caracterizaron a estas ciudades y, especialmente, los patrones de actividad que se observa en el registro arqueológico de estas urbes del pasado. 


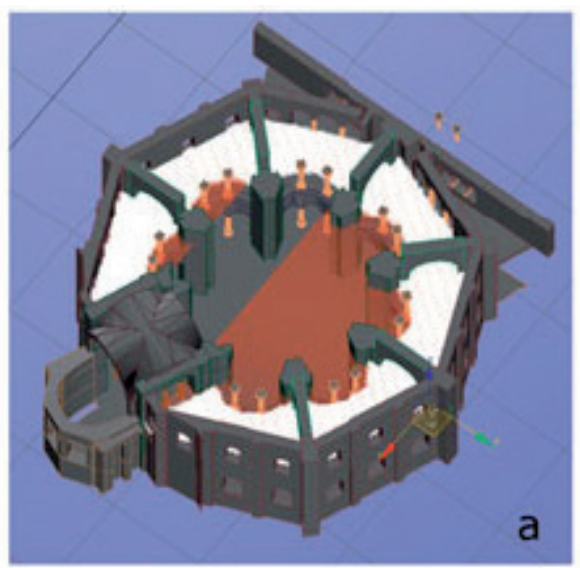

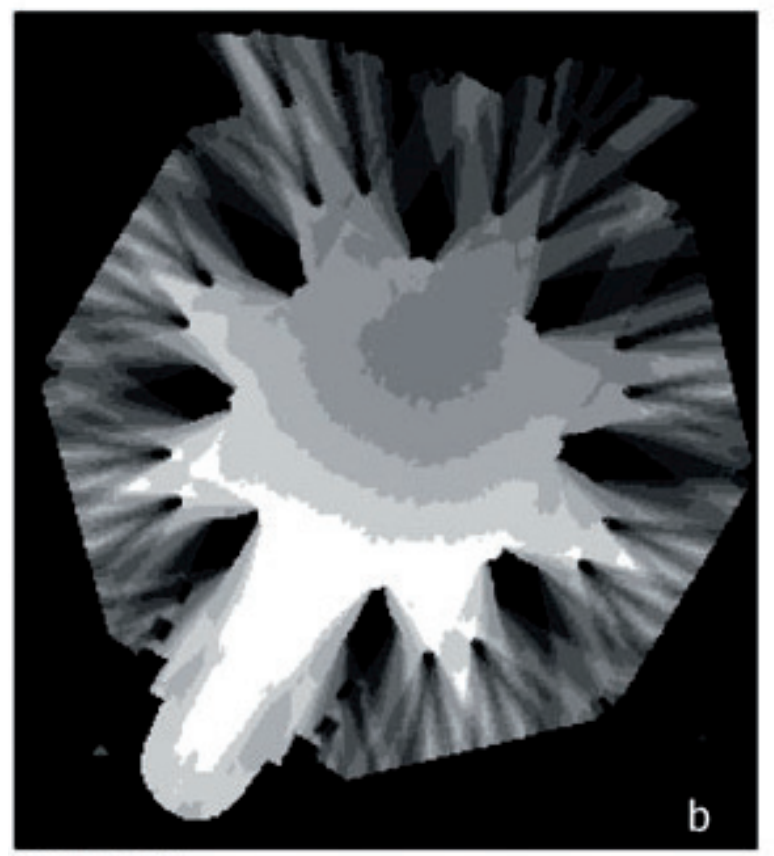

Times seen

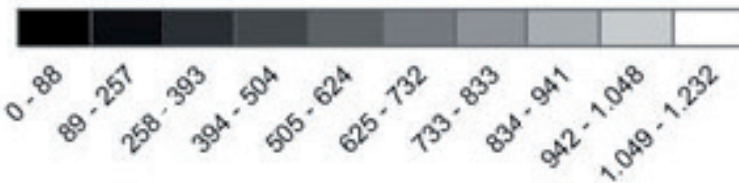

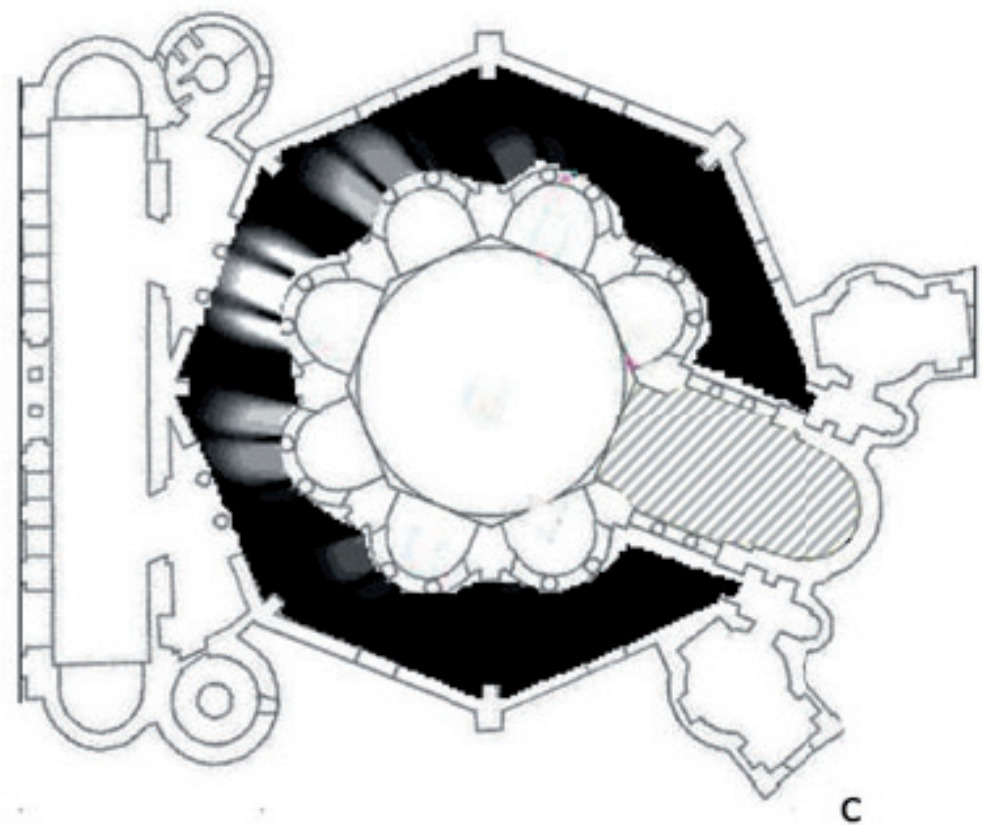

Visible area $\%$

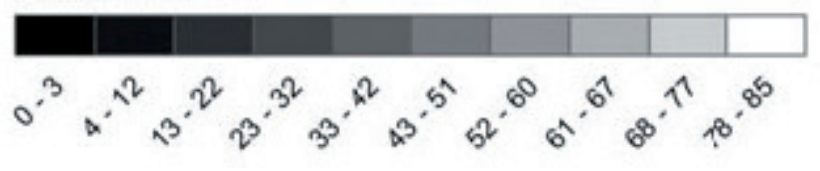

Figura 5. A) modelo 3D de San Vital de Rávena. B) gráfico de visibilidad en las que se aprecia las veces que el matroneum del edificio puede verse desde la planta baja. C) Gráfico de visibilidad en el que se aprecia el área visible del matroneum desde el cancel del edificio. A partir de Paliou 2014: Fig. 7.

Dentro de este grupo, los trabajos centrados en Pompeya pueden considerarse como un conjunto fundamental (cf. Laurence 2007; Grahame 2000; Weilguni 2011; Kaiser 2011; Laurence y Newsome 2011; Van Nes 2014b). En la ciudad vesubiana convergen dos factores fundamentales que explican esta preeminencia: la conservación de una compleja configuración urbana, con un porcentaje de superficie excavada muy elevado, y la posibilidad de conocer, a través del cotejo del registro arqueológico compilado a través de varias centurias de trabajos arqueológicos, la distribución de un gran número de actividades económicas y sociales acontecidas en la ciudad en el momento de su destrucción.

La publicación (con una segunda edición revisada) del célebre libro de Laurence (2007) sobre Pompeya puede considerarse como un hito fundamental en este 
sentido. Su pionero análisis de la trama urbana pompeyana sirvió para reconocer diversos patrones espaciales sobre la distribución de las actividades económicas en la ciudad (Laurence 2007: 62-81) en relación a los principales nodos de comunicación de la ciudad (Laurence 2007: 127). La comparación de los gráficos de accesibilidad de las casas de las regiones VI y VII, en relación al número de vanos que las comunican con las calles principales y al tamaño de las propiedades le ha permitido plantear una relación proporcional entre dichas variables y la RRA (Real Relative Asymetry) registrada en dichas insulae (Laurence 2007: 130133). Su trabajo, a pesar de haber recibido bastantes críticas (Taner 1995; George 1995; Ulrich 1997) puede considerarse como un trabajo seminal a la hora de considerar el análisis de todos los componentes de comunicación y viabilidad de una ciudad como objeto de análisis arqueológico.

Otro autor que ha afrontado más recientemente el estudio de la configuración urbana de las ciudades romanas como reflejo de su estructura social es Kaiser. Su obra, The Urban Dialogue (Kaiser 2000), fue uno de los primeros trabajos en aplicar este tipo de estrategias de análisis en contextos urbanos romanos diferentes a los del área vesubiana. Para ello recurrió a Emporion, un centro portuario en el que se desarrollaron un núcleo de población ibérico, un emporio griego y una ciudad romana, como caso de estudio. Su trabajo introdujo el análisis de profundidad (Depth) para el análisis de las calles y plazas de la ciudad, en tanto que ejes de circulación. Su método parte de asignar un valor de profundidad a cada calle en función del número de vías e interconexiones que había que cruzar desde las puertas exteriores hacía los diferentes componentes urbanísticos documentados en el trazado urbano registrado. Este trabajo sirvió para reconocer una configuración urbana en la que determinadas calles tenían una mayor relevancia dentro de la escala de hegemonía social de la ciudad. Además, fue el primer arqueólogo que conocemos que introdujo el método de la RRA (Real Relative Asymetry) para dotar de mayor inferencia estadística a sus inferencias.

Un segundo libro (Kaiser 2011) supuso la aplicación de esta misma perspectiva a un número más extenso de casos de estudio. Partiendo de la conceptuación funcional de las diversas categorías urbanísticas recogidas en la obra de Vitruvio, así como en otras referencias textuales (Kaiser 2011: 16-46), desarrolla su análisis de profundidad de las calles y demás unidades urbanísticas previamente definidas ${ }^{4}$ (fig. 6). En este caso, para justificar su análisis de profundidad toma dos tipos de referencia, las puertas de acceso y los recintos forenses del interior de todas las ciudades analizadas (Ostia, Pompeya, Silchester y Emporion). Los resultados obtenidos le permiten inferir la existencia de un patrón de circulación y distribución por el que cualquier habitante de una gran urbe romana podría reconocer inmediatamente cualquier calle o sector en orden a su importancia social dentro de la ciudad (Kaiser 2011: 199-205).

Otro ejemplo centrado en el análisis sintáctico espacial de la configuración urbana en época romana lo encontramos en un reciente estudio de Van Nes (2014b), de nuevo centrado en el caso de Pompeya. En cierto modo, su trabajo viene a confirmar algunas de las tesis planteadas por Laurence en su libro (2007). Por ejemplo, la aplicación de un análisis agent-based, generado mediante la herramienta correspondiente del software DEPTHMAP, ha permitido confirmar que la concentración de tiendas y negocios en la ciudad vesubiana era mayor en las calles con una mayor posibilidad de circulación (fig. 7). Su trabajo también destaca por mostrar la importancia de analizar la correlación de las dinámicas a microescala (edificios, insulae, barrios) con la red de circulación general, con objeto de conocer en mayor profundidad la evolución de la configuración urbana de las ciudades de la Antigüedad romana.

El empleo de este tipo de perspectivas de análisis para el estudio del urbanismo mesoamericano ha protagonizado recientemente en una serie de estudios (Seibert 2006; Morton 2007; Morton, Peuramaki-Brown, Dawson y Seibert 2012, 2014; Peuramaki-Brown 2012). La aplicación sistemática de los análisis de líneas axiales y de profundidad sobre diversos tipos de asentamientos prehispánicos como Teotihuacán (México), Naachtum (Guatemala) o Copán (Honduras), han permitido conocer hasta qué punto, la articulación de los rituales comunitarios que las elites urbanas utilizaban como elemento de naturalización del orden social, tenía también su reflejo en la propia estructura de urbanística de estas ciudades de época prehispánica. En concreto, el caso de Teotihuacán (Morton, Peuramaki-Brown, Dawson y Seibert 2012), representa mejor que ningún otro ejemplo mesoamericano esta materialización urbana de la ideología dominante a través de la distribución espacial de la liturgia en la propia planificación urbanística. El análisis axial planteado en Teotihuacán (fig. 8) refleja de forma gráfica hasta

\footnotetext{
${ }^{4}$ El uso social del espacio urbano en cada uno de los casos de estudio analizados en su obra pueden consultarse a través una web que sirve de suplemento a los datos desarrollados en el libro: http://faculty.evansville.edu/ak58/streets/
} 


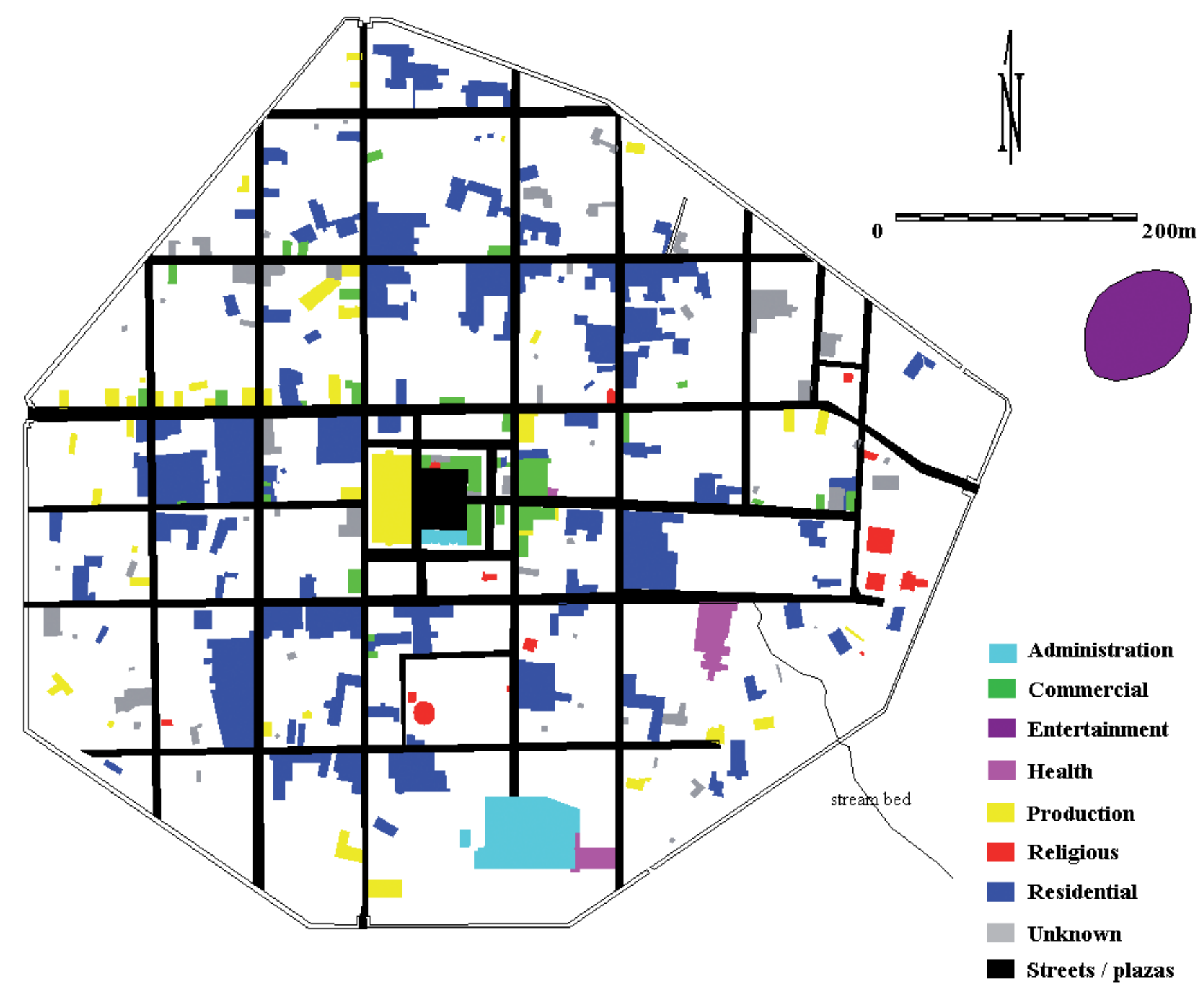

(C) Alan Kaiser 2011

Figura 6. Planimetría con los usos sociales del espacio urbano de la ciudad romana de Silchester (siglo IV d. C.). Según Kaiser 2011: http:// faculty.evansville.edu/ak58/streets/

qué punto el establecimiento de un eje vinculado a los principales centros de culto, sirve como articulador de la estructura urbana de la ciudad. No obstante, la más destacable novedad que ofrece el estudio sintáctico-espacial de este asentamiento se infiere a partir de la aplicación del análisis de profundidad a escala microespacial, sobre una serie de vecindarios de patio-central (Multi-Apartment Compounds) documentados en distintos sectores residenciales de la ciudad. Este parecido es utilizado por los autores del estudio (Morton, Peuramaki-Brown, Dawson y Seibert 2012: 391) para proponer que la configuración espacial de estos ámbitos residenciales se puede interpretar como un indicador del grado de aceptación de los aparatos ideológicos generados por las elites para naturalizar su preeminencia a través de la adaptación privada de la configuración espacial de los espacios de culto a escala local-vecinal.

Estos trabajos sobre la configuración urbana de Teotihuacán también han servido para establecer comparaciones con lo que sabemos sobre la distribución de actividades económicas en los distintos barrios de la urbe mesoamericana (Manzanilla 1996). Dicha comparación ha permitido comprobar la existencia de un patrón sintáctico de profundidad para la distribución funcional de los distintas unidades espaciales dentro de los barrios como Xoapan (Morton, Peuramaki-Brown, Dawson y Seibert 2012: figs. 3 y 4) (fig. 9). Los espacios relacionados con actividades productivas tienden a presentar valores de profundidad sintáctica mucho menores que los hábitats residenciales de las familias. 


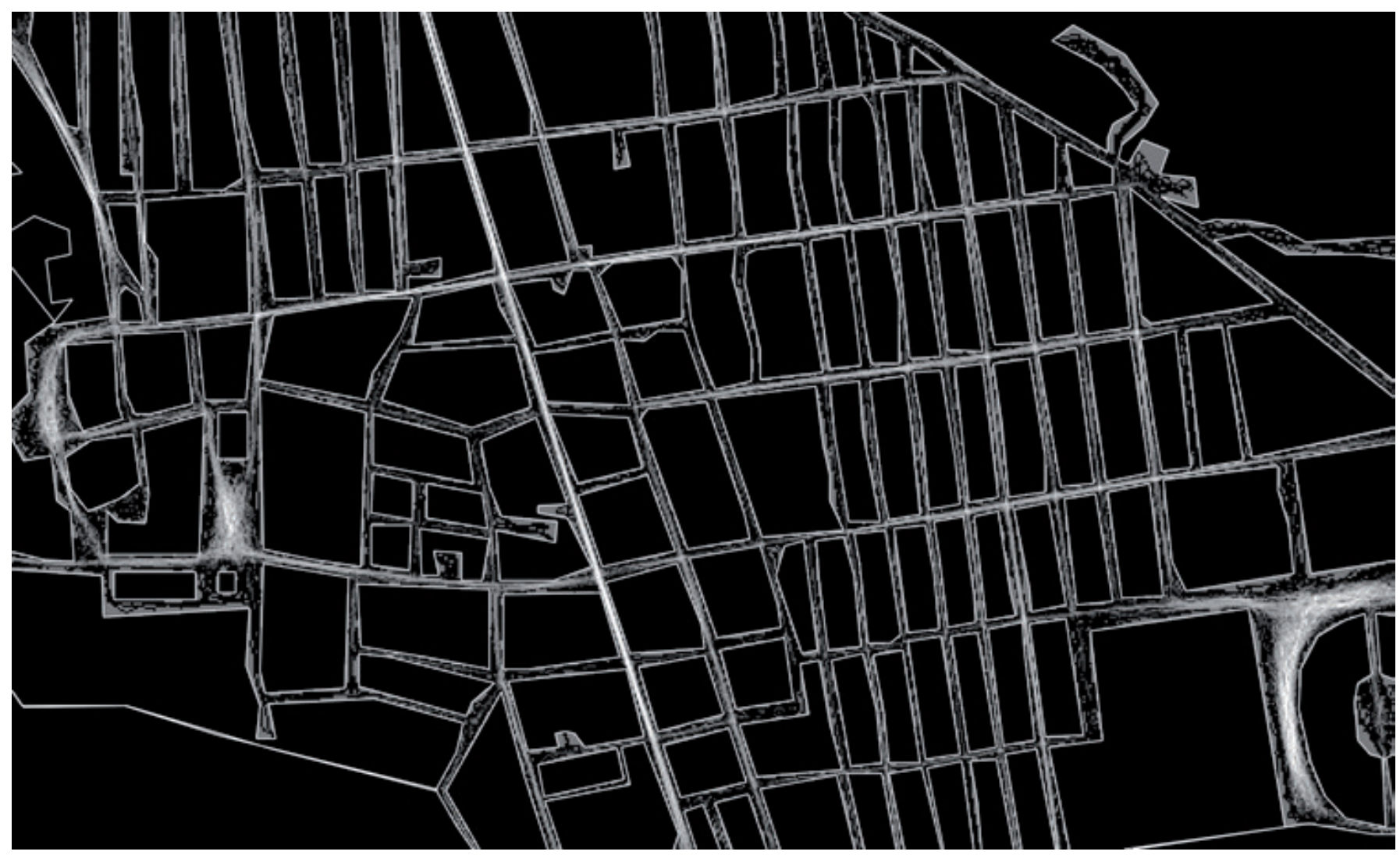

Figura 7. Modelo agent-based de los espacios públicos pompeyanos. Según Van Ness 2014: Fig. 9.

Desde un punto de vista comparativo, el contraste entre ambas perspectivas culturales, de los denominados periodos clásicos mediterráneo y mesoamericano, resulta un ejercicio muy ilustrativo. En ambos casos podemos ver la aplicación del principio de la economía del movimiento, explicitada en varios textos teóricos de Hillier y sus seguidores (Hillier 1996, 2002). No obstante, en términos específicamente culturales vemos diferencias entre las explicaciones manejadas en uno y otro contexto. En el caso mesoamericano, las explicaciones están basadas fundamentalmente en motivos de índole cosmológica o ritual. Sin embargo, las explicaciones preferentemente utilizadas para interpretar las inferencias sintáctico-espaciales de la configuración urbana de época romana se circunscriben, fundamentalmente, a principios de índole productiva o logística (Laurence y Newsome 2011). En el caso de la propia Urbs, a pesar de que contamos con algunas propuesta que han tratado de analizar la propia ciudad de Roma como una materialización simbólica de los aparatos ideológicos de las elites de época imperial (Wallace-Hadrill 2000; Zanker 2000; Carandini 2010), no tenemos constancia de ningún estudio sintáctico-espacial orientado a la documentación de este tipo de patrones.

\section{Geometría discreta de la ciudad antigua}

En relación al estudio empírico del urbanismo antiguo, una de las líneas de trabajo propuestas en los últimos años (Hillier 2014: 39-40) se refiere al análisis de las tramas geométricas que subyacen las ciudades a lo largo de su desarrollo, con objeto de conocer sus implicaciones en la vida cotidiana de las personas y grupos sociales.

A la concepción clásica del planeamiento urbanístico hipodámico, basado en la aplicación regular de un modelo de rejilla cuadrangular, representada en la planimetría de la antigua Mileto, Olinto o Priene, así como en múltiples escenarios urbanos contemporáneos (fig. 10), algunos teóricos del urbanismo han contrapuesto el concepto de ciudad orgánica (Hillier 2002; Carvalho y Penn 2004), como aquella generada a lo largo de un proceso histórico en el que no se ha podido aplicar un plan de diseño urbanístico unitario a lo largo del tiempo.

Con el objetivo de testar la posible existencia de un principio geométrico discreto general para la configuración urbana de estas ciudades orgánicas, el propio Hillier y algunos de sus colegas han analizado (con la ayuda de las herramientas de análisis axial y de profundidad del 
software DEPTHMAP) un gran número de ciudades orgánicas desarrolladas sin aparentes conexiones culturales (Hillier y Vaugan 2007). El resultado de estos trabajos es que un número sorprendentemente elevado de estas ciudades (con desarrollos históricos tan diferentes como Londres, Tokio o Nicosia) muestran un patrón geométrico común, el llamado Deformed Wheel Model. (fig. 11).
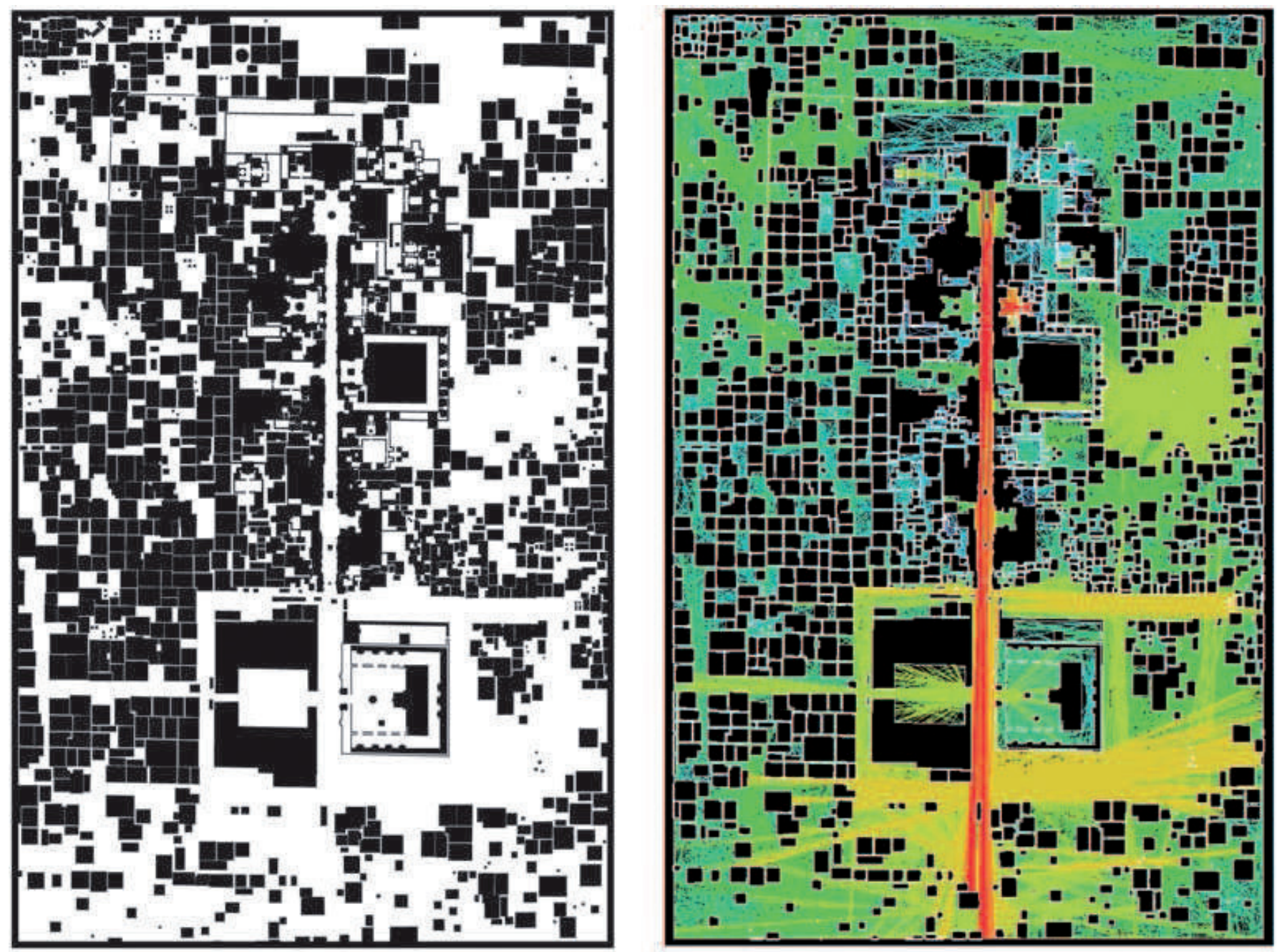

Figura 8. Plano de la trama urbana del centro de Teotihuacan y mapa axial. Según Morton, Peuramaki-Brown, Dawson y Seibert $2012:$ Fig. 5.

a

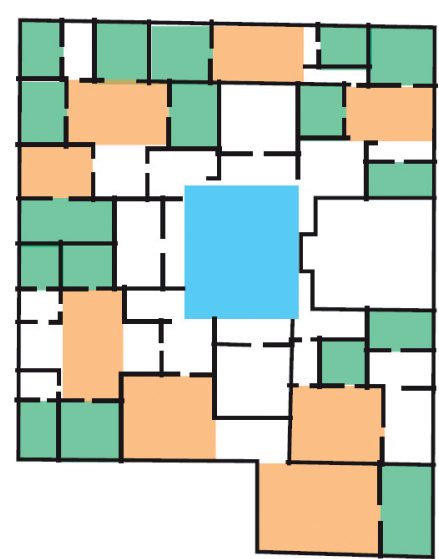

b

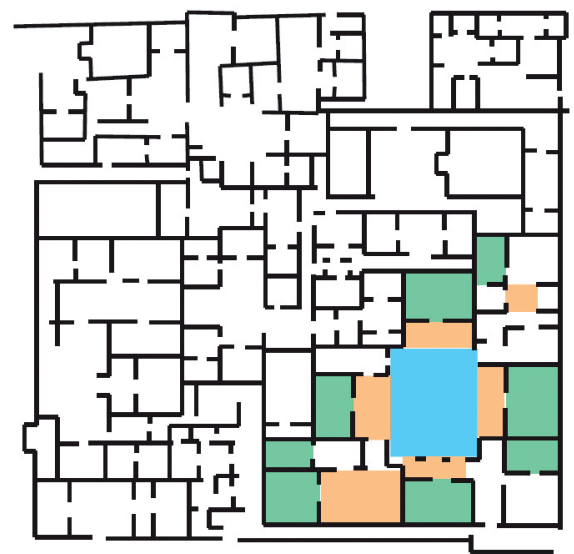

C

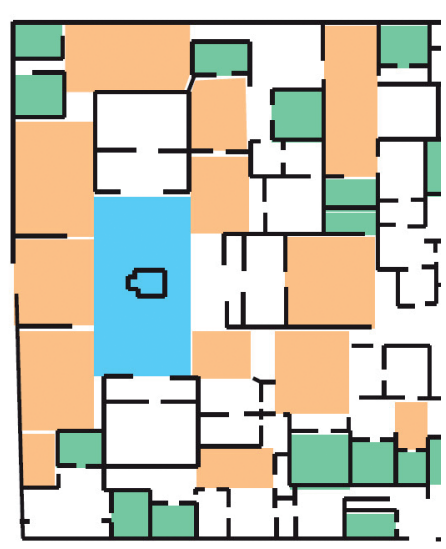

Figura 9. Planimetría esquemática de tres multi-apartment compounds de Xoalpan (Teotihuacan). En azul se destacan los patios centrales con indicios de culto a los ancestros. En naranja aquellos espacios relacionados con áreas productivas. En verde aquellos espacios relacionados con el hábitat residencial de las familias. Según Morton, Peuramaki-Brown, Dawson y Seibert 2012: Fig. 3. 


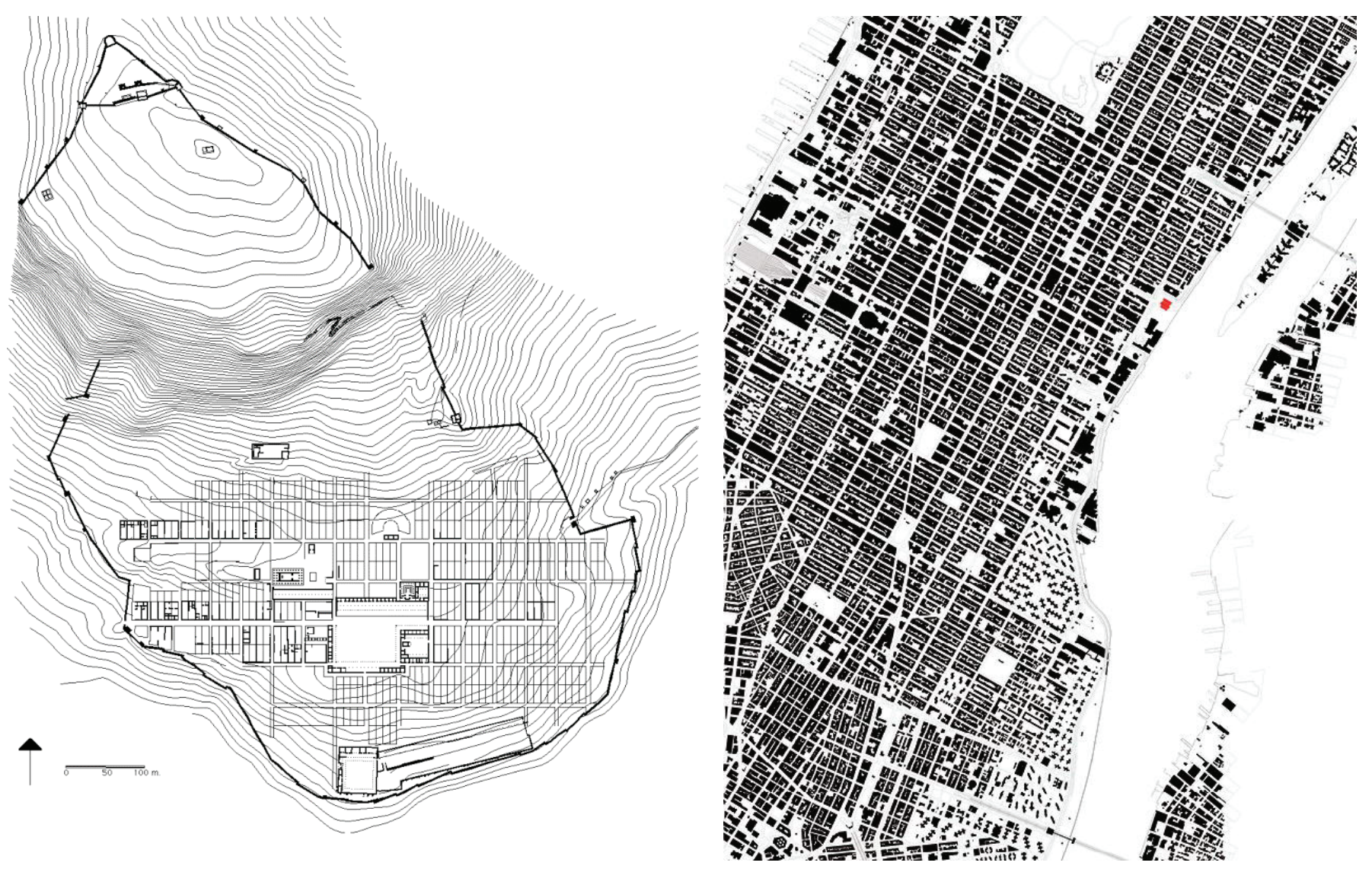

Figura 10. Izquierda. Plano Esquemático de la trama urbana de Priene (Asia Menor) en época helenística (Fuente: Wikipedia). Derecha. Plano esquemático de la retícula urbana del Middle/Lower Side de Manhattan (Nueva York) (Fuente: web de la ONU).
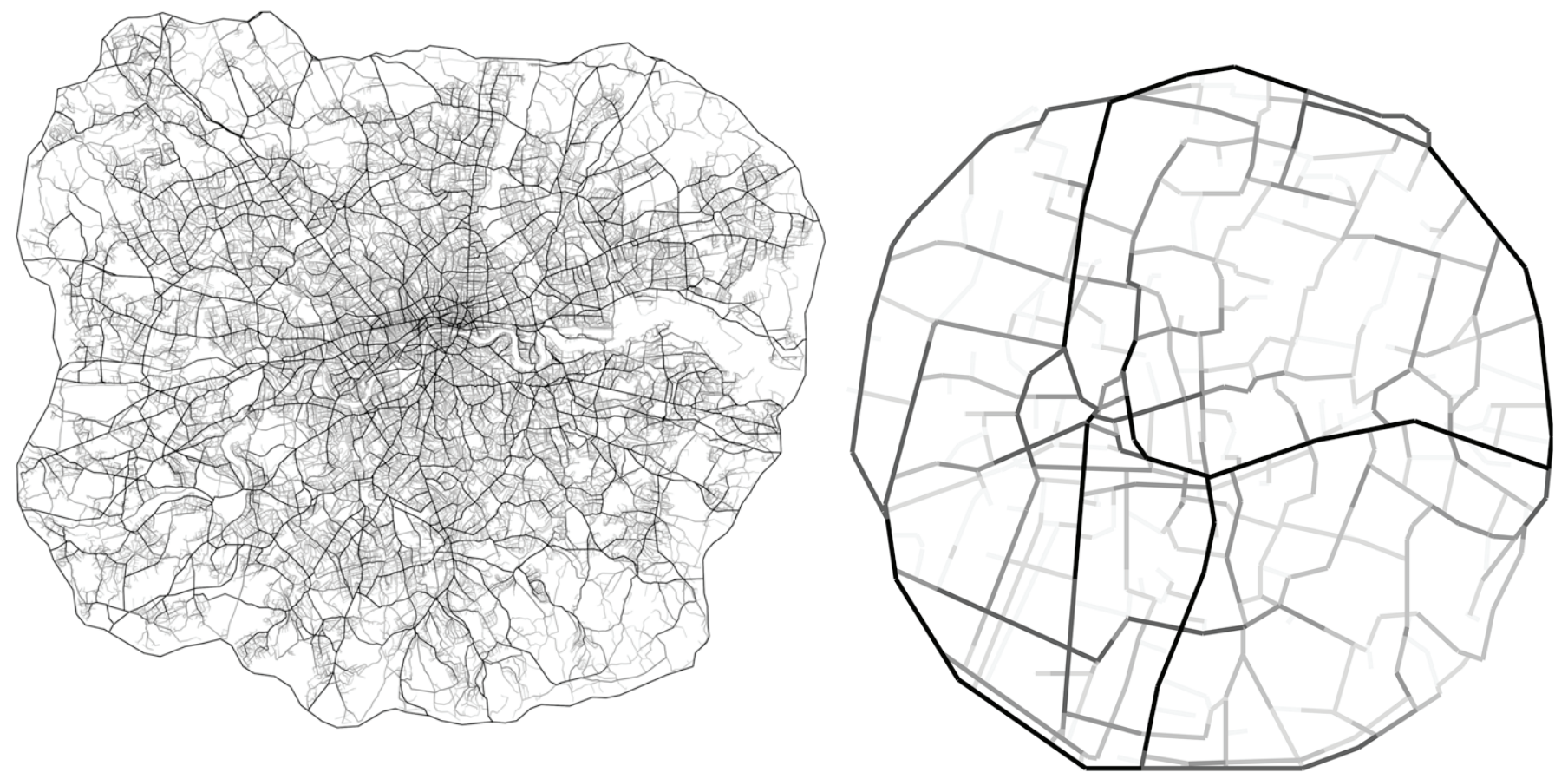

Figura 11. Izquierda. Mapa axial con las principales vías de comunicación del área metropolitana de Londres. Derecha. Mapa axial con las principales vías de comunización del centro histórico de Nicosia (Chipre). A partir de Vaughan y Hillier 2007. 
Este modelo consiste en la generación de una rejilla con forma de rueda de bicicleta con radios irregulares configurados por el propio desarrollo urbanístico. Esto genera una cuestión fundamental: ¿Cómo es posible que una misma trama geométrica pueda emerger tras décadas o siglos de actividad urbanística de innumerables agentes y contextos sociales, económicos y culturales tan diversos?

Tal vez, uno de los casos de estudio arqueológico más adecuados para testar esta hipótesis sea la propia Roma. Una comparación de la disposición topográfica de la Urbs en época imperial en relación con la distribución de las principales vías de circulación del actual área metropolitana romana (fig. 12: 1 y 2), parece mostrarnos que el desarrollo histórico de la ciudad antigua, fosilizado en la disposición de las principales vías radiales y anulares de comunicación, parece reflejar la configuración del modelo geométrico del DWM (Deformed Wheel Model) ya desde la Antigüedad.

La esencia del modelo DWN, como patrón geométrico discreto en la configuración de las ciudades orgánicas, se basa en el concepto de centralidad. Varios estudios han resaltado la importancia topográfica de los ámbitos centrales como elementos fundamentales de la configuración metropolitana en distintos contextos urbanos de la Antigüedad, no sólo romana (Kaiser 2011), sino también en otros ámbitos culturales como el Oriente próximo o Mesoamérica (Smith 2003; Collins 2005). No obstante, muchas de estas conclusiones sobre la geometría discreta de las ciudades arqueológicas se pueden alcanzar sin la necesidad de la aplicación específica de herramientas de sintaxis espacial (Smith 2011: 176). De hecho, tenemos diversas noticias históricas que nos hablan de configuraciones urbanas relativamente similares. Uno de los casos más conocidos está recogido en las Leyes platónicas (VIII, 845 b-c), donde tenemos la descripción de una ciudad utópica, conocida como Magnesia, cuyo diseño urbanístico se asemeja al ideal pitagórico de la rueda con radios y distritos concéntricos (Cervera 1976: fig. 2).

A la vista de estas pertinentes críticas, creemos que la verdadera innovación que ofrece la aplicación de este tipo de estrategias analíticas para el cotejo de los patrones geométricos emergentes en la configuración de las ciudades (no sólo arqueológicas) se refiere a la capacidad de ilustrar las consecuencias sociales de las tramas urbanísticas en términos de segregación. Para ilustrar esta afirmación vamos a volver al tema de las ciudades de planta hipodámica.

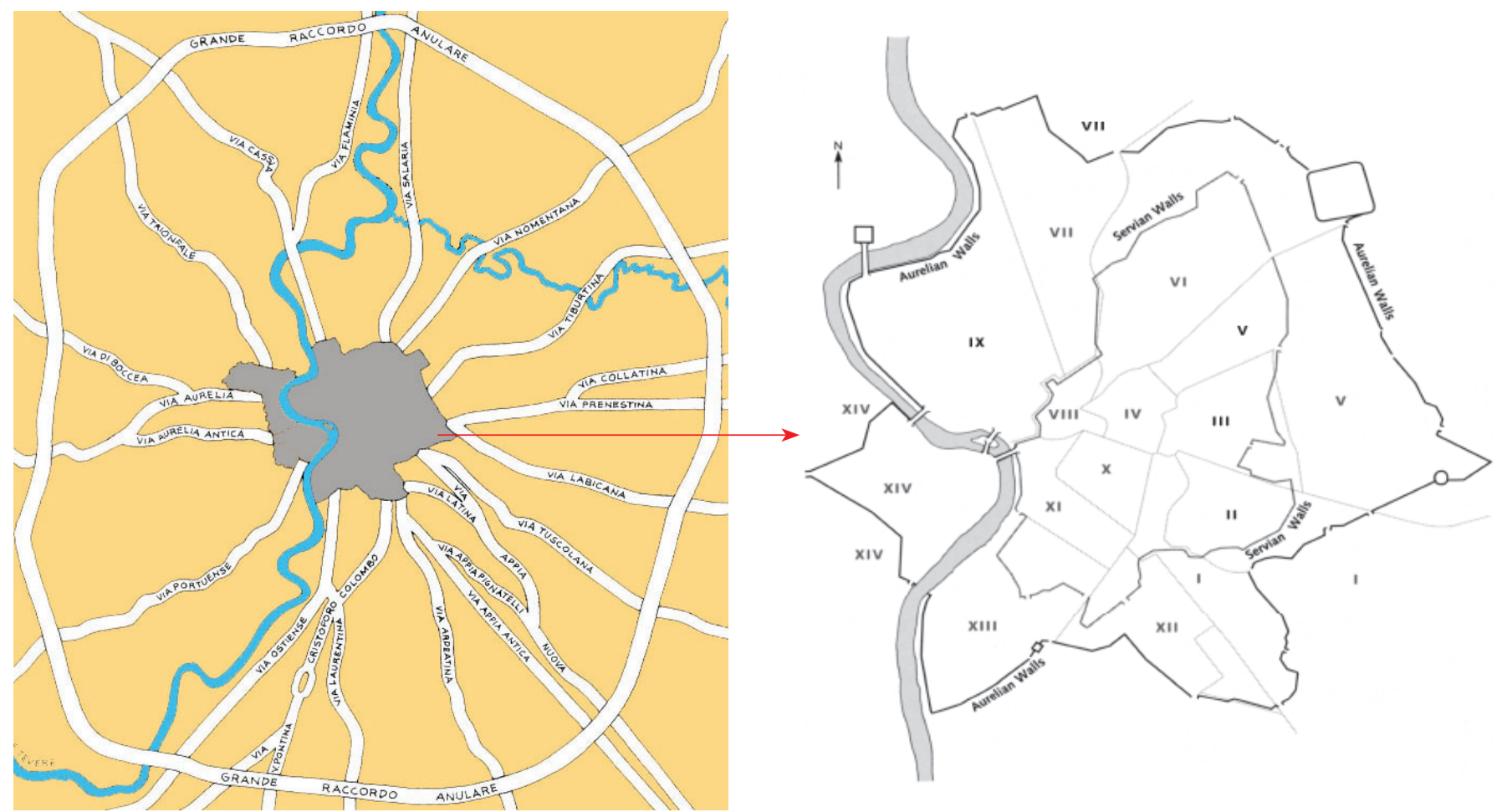

Figura 12. Izquiera. Plano del trazado urbano de las principales vias de comunización en el area metropolitana romana dentro del GRA . Derecha plano esquemático del área urbanizada de la Roma imperial en el controno de la Muralla Serviana y la Muralla Aureliana (Siglo IV d. C.). A partir de Jolivet, Pavolini, Tomei, y Volpe 2009: (generale) y Coarelli 2008: Fig. 2. 
A diferencia de las llamadas ciudades orgánicas, las ciudades de planta hipodámica están dotadas de una estructura geométrica con forma de rejilla ortogonal. Frente a la idea de concepción jerarquizada de la centralidad, el diseño geométrico de la planta hipodámica ha sido interpretado por algunos autores como reflejo de una cierta tendencia isonómica en la que todos los componentes de la trama urbana quedan aparentemente equiparados. En este principio de diseño urbanístico se ha querido ver un reflejo del ideal democrático de la polis clásica (Hoepfner y Schwandner 1994), del que el propio Hipódamo de Mileto fue un teórico destacado (Cervera 1987). Sin embargo, la aplicación de herramientas Space Syntax para el análisis de ciudades o barrios diseñados a partir de la aplicación de este urbanismo ortogonal, han permitido mostrar que, a pesar del aspecto aparentemente igualitario de las tramas urbanísticas, este tipo de forma urbana también genera ámbitos nucleares y áreas de segregación (fig. 13).

El análisis sintáctico-espacial de la trama urbana (obtenida a partir de la aplicación de un programa sistemático de teledetección) de la ciudad helenístico-romana de Dura-Europos (Siria) por parte de Benech (2010) nos ha mostrado de qué modo la implantación de un diseño hipodámico, basado en una rejilla ortogonal de manzanas regulares, en realidad genera un patrón de segregación espacial de determinados sectores de la ciudad (Benech 2010: 408). Estos resultados, obtenidos mediante la aplicación de la herramienta de análisis de visibilidad de DEPTHMAP, parecen refrendar la tesis planteada por Sennett en un célebre trabajo (Sennett 2004) sobre las raíces ideológicas de la trama ortogonal en las ciudades modernas. Según el sociólogo norteamericano, detrás de la aparente racionalidad isonómica que parece guiar este tipo de organización urbana, se esconde el deseo de las elites rectoras de implantar un orden coercitivo, reflejado en la segregación forzosa de comunidades marginadas, para controlar más fácilmente a diversos sectores urbanos. Este principio de coerción urbanística, a la vista de los datos atisbados en el trabajo de Benech (2010), también habría podido registrarse en el diseño urbano de ciudades del periodo helenístico y romano.

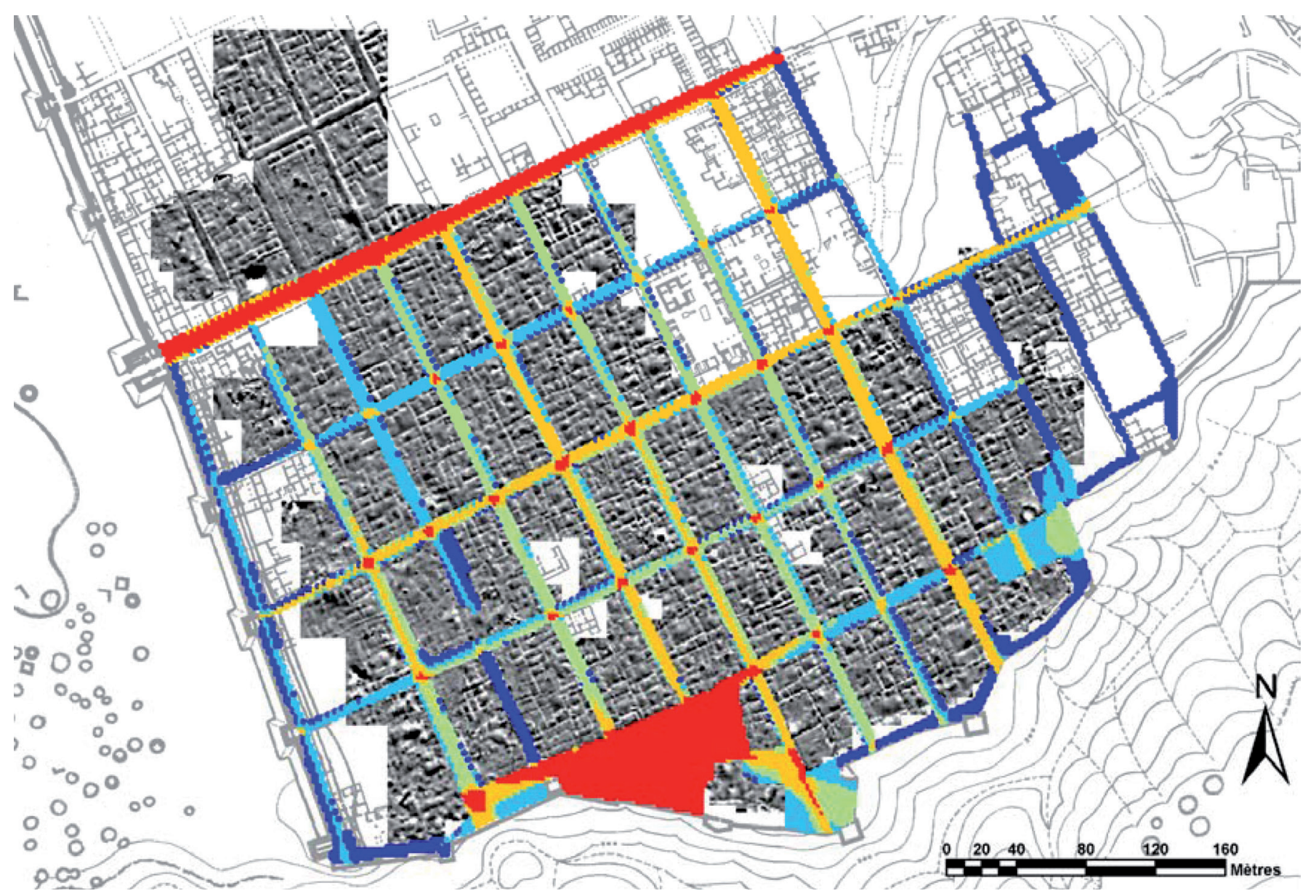

Figura 13. Gráfico de visibilidad de la red urbana en la antigua Dura-Europos (Siria). El color expresa la escala de conexión visual (máximo rojo/mínimo azul). Según Benech 2010: Fig. 5. 


\section{La lógica social del paisaje}

La aplicación de herramientas de sintaxis espacial para el análisis de la configuración de paisajes históricos y prehistóricos se ha desarrollado, muy recientemente, en paralelo al surgimiento de concepciones alternativas para el urbanismo en las sociedades antiguas (Smith 2007; Isendhal y Smith 2013). Sintetizando en gran medida estas discusiones, del mismo modo que nos hemos referido a la existencia de ordenamientos geométricos discretos en ciudades llamadas orgánicas, la aplicación de técnicas de sintaxis espacial también se ha utilizado como método para identificar patrones de configuración paisajística en diversos tipos de asentamientos.

La mayor parte de estos trabajos se basan en una aplicación previa de sistemas de información geográfica para el análisis territorial de las rutas menos costosas (least-cost paths analysis5) para visualizar la red de comunicaciones internas dentro de regiones o asentamientos (Anderson 2012). En un trabajo desarrollado por Neff (2010), la red de rutas menos costosas del Cerro Jazmín, un asentamiento prehispánico en alto con una ocupación dilatada desde el periodo Formativo hasta su etapa de máximo desarrollo durante la época Post-clásica (Pérez, Anderson y Neff 2011), ha sido utilizada como base para la aplicación de un análisis sintáctico de profundidad entre los denominados nódulos de circulación, coincidentes con las casas y los aterramientos agrícolas distribuidos por toda la superficie de este cerro amesetado. Esta aplicación le ha permitido documentar un ordenamiento urbanístico interno con un patrón sintáctico espacial claramente articulado (fig. 14) en un asentamiento considerado, en un principio, como un hábitat disperso sin un orden generalizado.

En esta misma línea de aplicación mixta de SIG y sintaxis espacial, dos trabajos (Hudson 2012; RichardsRisseto 2012) publicados en un volumen sobre la aplicaciones de análisis LCP en Arqueología, son dos claros ejemplos de las posibilidades de este tipo de línea de investigación. Ambos trabajos son claras representaciones de cómo, la aplicación de herramientas de sintaxis espacial para el análisis puede servir para visualizar distintos tipos de dinámicas sociales ocultas tras la configuración espacial de paisajes sagrados y funerarios a escala territorial.

\footnotetext{
5 Para una introducción sobre este tipo de análisis espacial cf. White y Surface-Evans 2012; Herzog 2014.
}

\section{Arqueología de las arquitecturas (ocultas) del poder}

Dentro de este punto vamos a incluir todos aquellos trabajos que han utilizado herramientas tipo Space Syntax con objeto de registrar el modo en que el espacio arquitectónico ha sido utilizado por diversos poderes para imponer determinados principios ideológicos y sociales sobre las poblaciones que gobiernan. A diferencia de otros enfoques tradicionales, centrados en el estudio de arquitecturas monumentales con evidentes connotaciones propagandísticas, este grupo de trabajos ha tomado como foco de atención principal sobre edificios funcionales, muchas veces vinculados a la vida cotidiana de las personas, así como aquellos complejos arquitectónicos relacionados con diversos aparatos administrativos de los Estados desde una perspectiva histórica.

El análisis sintáctico-espacial de edificios de uso rutinario (como las casas, las escuelas, hospitales o prisiones) ha sido utilizado por algunos autores para registrar el desarrollo de mecanismos biopolíticos con los que los poderes rectores regulan ciertos aspectos de la vida cotidiana y la conducta de sus súbditos (como la educación, la sexualidad, las formas de estructura familiar y de las unidades domésticas, etc.).

Precisamente este tema fue objeto de un libro recientemente publicado por quien suscribe este artículo (Bermejo 2014). Dicho trabajo se centra en el análisis del impacto de las políticas sociales desarrolladas por el estado romano en la vida cotidiana de las comunidades locales de Meseta oriental hispana. La aplicación de análisis sintáctico-espaciales sobre los diversos ejemplos de arquitectura doméstica registrados por la Arqueología en la región nos permitió comprobar que la configuración espacial de algunas de estas casas respondía a las necesidades y restricciones sociales del modelo familiar configurado por la normativa augustea. Dicho modelo, fue codificado por diversos aparatos del Estado como el derecho privado, la política fiscal o los textos de autores clásicos claramente vinculados al poder imperial, cuyo ejemplo más relevante en lo que se refiere a la arquitectura se puede encontrar en el libro VI de los tratados vitruvianos. Pero, al mismo tiempo, el análisis sintáctico-espacial de otros ejemplos de la muestra seleccionada nos permitió comprobar que en otras ocasiones la configuración espacial de la casa podían interpretarse como un prueba fehaciente de la resistencia cotidiana de parte de estas comunidades (claramente desvinculadas de las elites en la escala social local) a someterse a la normativa imperial de ordenación de la vida familiar. 


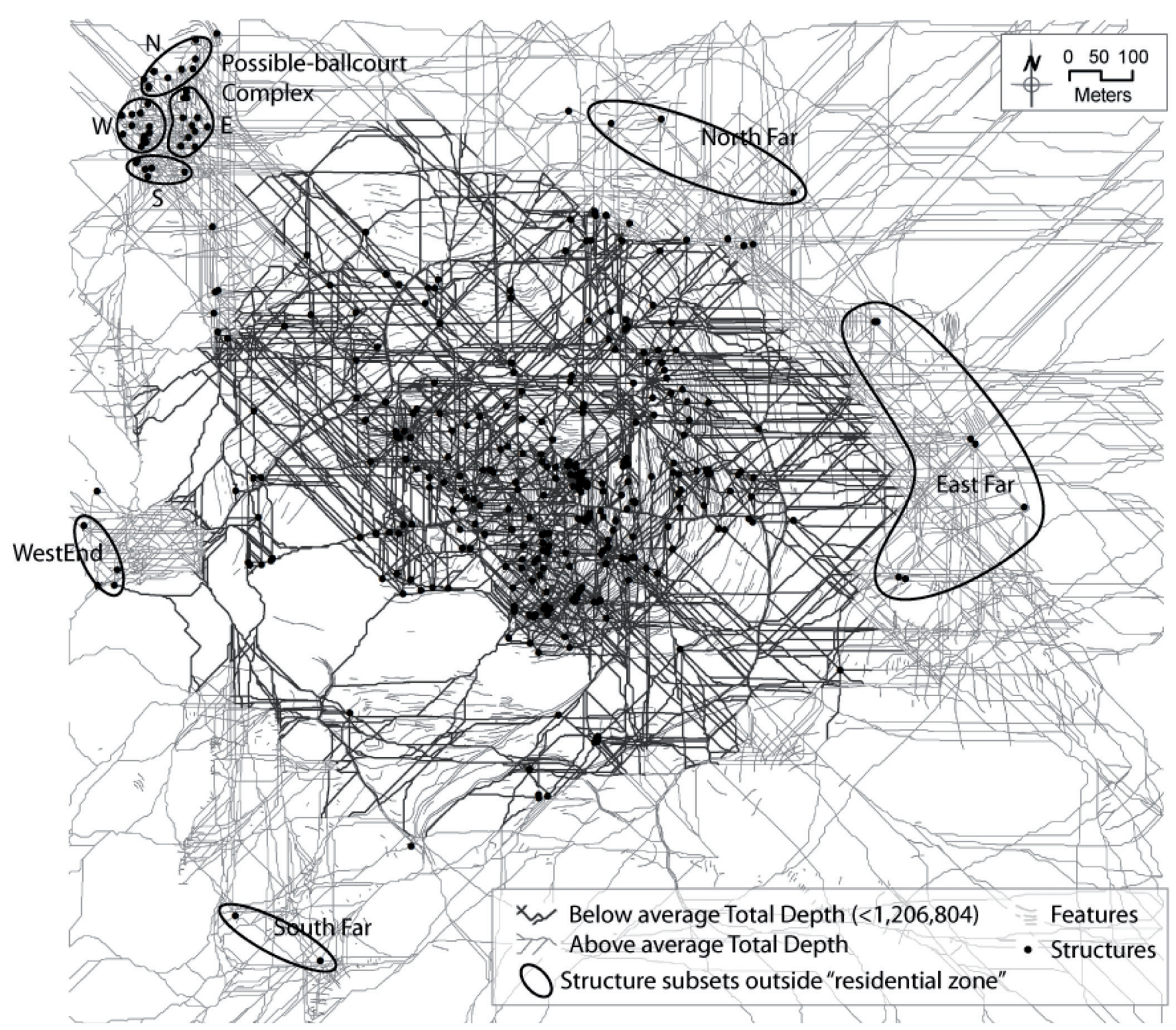

Figura 14. Gráfico de profundidad global (incluyendo los datos del análisis space syntax más los del least cost path) del área residencial del asentamiento prehispánico del Cerro Jazmín (Oaxaca, México). A pesar de la orografía complicada del terreno, es posible reconocer un patrón de distribución espacial entre los asetamientos aterrazados (márcados con un punto). Según Neff 2010: Fig. 5.15.

Existen otros autores, circunscritos casi siempre al ámbito de la Arqueología histórica, que se han encargado de estudiar este tipo de problemáticas en edificios pertenecientes a diversos contextos temporales y culturales. Es el caso por ejemplo de A. Zarankin, un arqueólogo que ha aplicado estrategias de análisis tipo Space Syntax para el estudio del diseño arquitectónico de edificios públicos y privados de la Argentina entre los siglos XIX y XX (Zarankin 1999, 2002; Funari y Zarankin 2003; Zarankin y Niro 2006). La aplicación comparativa de análisis de accesibilidad, asimetría relativa, valor de control y los índices desarrollados por Blanton (1994:
31-36) ha sido desarrollada por este autor para registrar la implantación de estructuras hegemónicas propias de las sociedades capitalistas a través del diseño arquitectónico de espacios como bancos, escuelas públicas y casas construidas en el entorno bonaerense. Especialmente destacables son sus trabajos en relación a los centros de represión clandestina de la dictadura militar argentina (1978-1983) (Zarankin y Niro 2006). A través de ellos hemos podido reconocer una serie de patrones espaciales propios de estos centros diseñados para conseguir de forma más efectiva la confusión y coerción de los prisioneros políticos torturados en estos centros (fig. 15). 


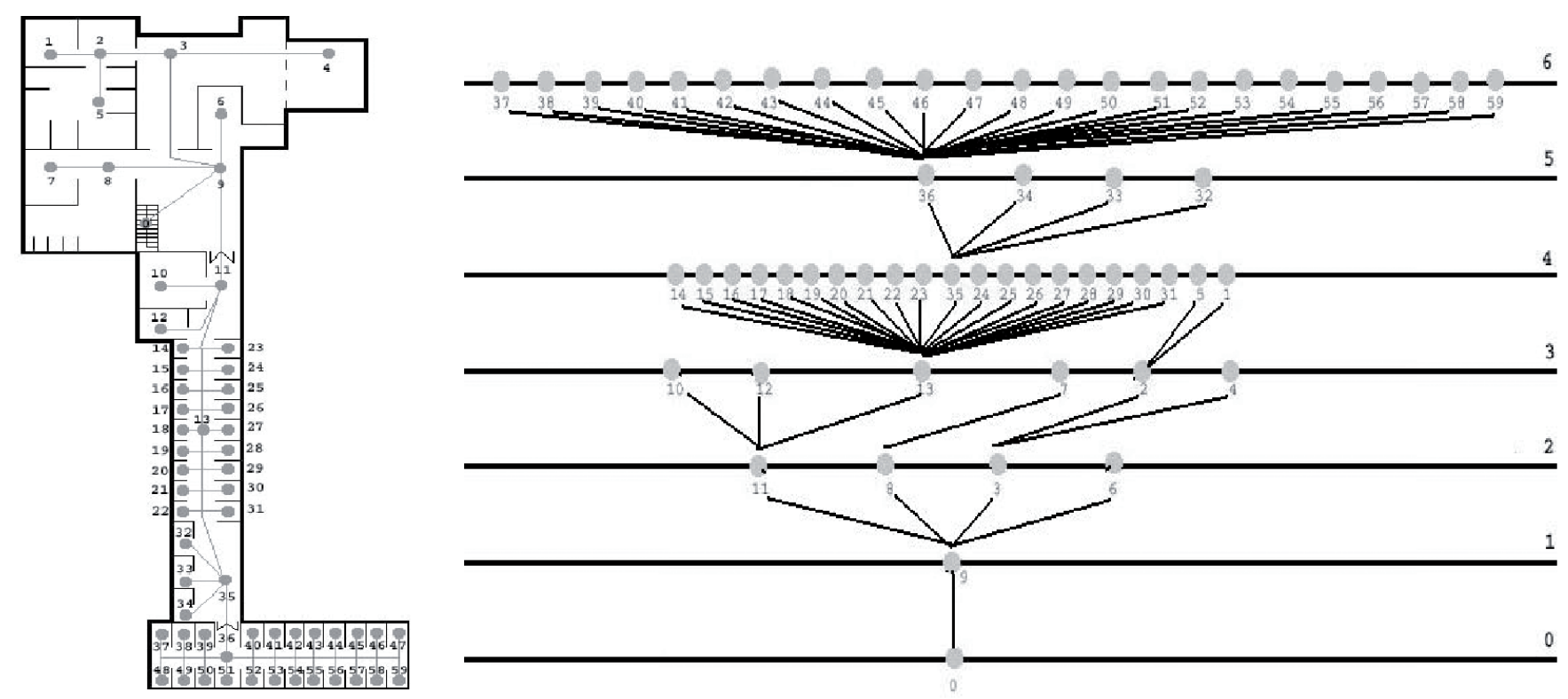

Figura 15. Gráfico de accesibilidad del Centro Clandestino de Detención de la dictadura militar argentina "Club Atlético" también denominado como "Centro Antisubversivo"(Buenos Aires, Argentina). Según Zarankin y Niro 2006: Fig. 4.

\section{Tendencias presentes y perspectivas de futuro}

Desde un punto de vista historiográfico hemos de destacar viarias tendencias que se han acentuado en los últimos años. Una de ellas es la gran concentración de aplicaciones sintáctico-espaciales en contextos de época romana. Como ya hemos mencionado, en gran parte, esta tendencia puede explicarse por la posibilidad específica que ofrecen algunos yacimientos romanos para analizar ciudades complejas excavadas en extensión, sobre todo en relación a periodos anteriores y posteriores. Sin embargo, esta especial concentración de aplicaciones resalta bastante en contraste con un marco historiográfico, el de la Arqueología clásica continental, que en las últimas décadas ha sido muy poco dado a liderar innovaciones de corte teórico-metodológico en comparación con otras tradiciones arqueológicas a escala global.

Otros patrones historiográficos que se registran en la bibliografía referida muestran diferentes orientaciones epistemológicas en clave geográfica. Por un lado, gran parte de los estudios desarrollados por autores anglosajones (a los que habría que añadir de forma destacada las investigaciones desarrolladas en el marco de centros holandeses como Leiden) se caracterizan por plantear sus estudios desde una perspectiva esencialmente inferencial, cuando no meramente descriptiva. De otra parte, los investigadores iberoamericanos se caracterizan por presentar puntos de vista mucho más inductivos, en los que la aplicación de técnicas sintáctico-espaciales se concibe como una mera herramienta que debe estar orientada por concepciones teóricas independientes y previamente ajustadas al contexto histórico objeto de estudio.

En ambas corrientes, que en ningún caso pueden considerarse como ámbitos estancos, detectamos ciertas tendencias perniciosas que queremos resaltar a modo de crítica constructiva. En el caso de los estudios descriptivistas, es relativamente frecuente encontrar trabajos sin un planteamiento explícito de hipótesis teóricas previas. En muchos casos, el esbozo de un marco interpretativo se produce después de la obtención y el tratamiento de los datos. Esto supone cierta querencia por el planteamiento de hipótesis post hoc, en función de los datos manejados. De esta manera se generan interpretaciones arqueológicas que, obviamente, no pueden ser falsadas pero que al mismo tiempo son incapaces de aclarar problemas históricos o procesos sociales de importancia. La cara opuesta a esta tendencia la encontramos en algunos trabajos del ámbito hispano en la que el planteamiento de un aparato conceptual muy rígido configura un tratamiento selectivo de los datos, obviando aquellos que no concuerdan con las ideas inicialmente planteadas. 
Para concluir este trabajo quisiéramos enunciar dos elementos que, desde nuestra óptica, habrán de tener importancia en el desarrollo futuro de este tipo de aplicaciones en el marco de la Arqueología de la Arquitectura a escala global. El primero de ellos se refiere al planteamiento sistemático de proyectos de investigación comparativa desde una perspectiva intercultural. A lo largo de estas líneas hemos comprobado que la aplicación de las mismas herramientas e índices sintático-espaciales para el análisis arqueológico de edificios y ciudades de los más diversos ámbitos culturales. Sin embargo, en gran parte debido a la inercia de la especialización académica, son muy pocos los trabajos que afronten la comparación de los datos obtenidos en contextos históricos diferentes de un modo consistente y sistemático (quizá la mayor excepción la encontremos en el libro de Blanton 1994). Es cierto que, como ya hemos mencionado, en los últimos años hemos asistido al surgimiento de encuentros y a la elaboración de libros editados destinados a la publicación de aplicaciones de Space Syntax de estudios de diversos contextos culturales. Pero hemos de reconocer que casi siempre se trata de estudios compartimentados en sus respectivos contextos históricos o culturales. Un reto de futuro en el marco de este tipo de aplicaciones arqueológicas, será el de desarrollar proyectos científicos orientados programáticamente (Smith y Peregrine 2012; Feinman 2012) desde una perspectiva comparativa sistemática.

Otra línea de trabajo que habrá de ser abordada en los próximos años, en clara relación con el desarrollo de estudios comparativos, se refiere a la cuestión de la estandarización de los datos generados en aplicaciones arqueológicas de este tipo de herramientas de análisis. Por sus propias características, la generación de datos analíticos en el marco de estudios tipo Space Syntax se ajusta perfectamente a un alto nivel de estandarización matemática, lo que genera un marco de actividad especialmente recomendable en términos de inferencia estadística. Al mismo tiempo, el acceso generalizado a aplicaciones software (como DEPTHMAP) ofrece un entorno ideal para el uso estandarizado de índices gráficos en estudios de este tipo. Sin embargo, hasta la fecha, son muy pocos los investigadores que se hayan aventurado a ofrecer los archivos originales con los datos aritméticos y gráficos de sus análisis, a pesar de que el número de publicaciones científicas sobre este tipo de aplicaciones está aumentando. Entre las razones fundamentales que encontramos para explicar esta situación divergente debemos destacar aquellas relacionadas con la percepción y la valoración de los méritos académicos relacionados con la publicación científica en Arqueología. En la actualidad, la escasa o nula valoración de la publicación científica de los datos generados en un formato telemático (bien sea a través de bases de datos, bien a través de archivos gráficos de imágenes o generados a través de diversos tipos de aplicaciones informática como SIG, etc.) ayuda a mantener esta situación en la que, en la mayoría de los casos la publicaciones textuales se producen sin que los lectores o investigadores interesados puedan tener acceso a los datos en los que están basados.

A la vista de lo que está ocurriendo en otras áreas de la investigación científica actual, en las que el desarrollo de inferencias establecidas sobre grandes repertorios de datos (Big Data) está revolucionando nuestra capacidad analítica en muchos aspectos, creemos necesaria una reconsideración estratégica de las políticas de valoración de méritos científicos en el ámbito de la Arqueología actual. En este sentido, las posibilidades analíticas que se han esbozado en el marco de las aplicaciones arqueológicas de herramientas de sintaxis espacial, podrán jugar un papel importante en un deseable proceso de convergencia y diálogo entre la Arqueología y otras ciencias sociales como el Urbanismo, la Economía, la Historia, la Sociología, la Geografía humana y la Arquitectura en su más amplia acepción.

\section{BIBLIOGRAFÍA}

Al-Sayed, K.; Turner, A.; Hillier, B.; Iida, S. y Penn, A. 2014: Space Syntax Methodology. Burtlett School of Architecture. UCL. Londres.

Anderson, D. 2012: "Least Cost Pathway Analyses in Archaeological Research: Approaches and Utility". En D. A. White y S. L. Surface-Evans (eds.): Least Cost Analysis of Social Landscapes: Archaeological Case Studies. University of Utah Press. Salt Lake City, pp. 239-257.

Bafta, S. 2003: "Space Syntax: A Brief Introduction to its Logic and Analytical Techniques". Environment and Behavior 35, pp. 17-29.

Benech, Ch. 2010: "The use of "space syntax" for the study of city planning and household from geophysical maps: the case of Dura-Europos (Syria)". Städtisches Wohnen im östlichen Mittelmeerraum 4. Jh. v. Chr.-1. Jh. n. Chr. Archäologische Forschungen 18, pp. 403-416.

Benedikt, M. L. 1979: "To take hold of space: isovists and isovist fields". Environment and Planning B: Planning and Design 6, pp. 47-65.

Bermejo Tirado, J. 2009: "Leyendo los espacios: una aproximación crítica a la sintaxis espacial como herramienta de análisis arqueológico". Arqueología de la Arquitectura 6, pp. 47-62.

Bermejo Tirado, J. 2013: "Análisis social de la arquitectura doméstica en la región del Alto Duero: una aproximación sintáctico-espacial”. En S. Gutiérrez e I. Grau (eds.): De la estructura doméstica al espacio social. Lecturas arqueológicas del uso social del espacio. Servicio de publicaciones de la Universidad de Alicante. Alicante, pp. 141-154.

Bermejo Tirado, J. 2014: Arqueología biopolítica. La sintaxis espacial de la arquitectura doméstica romana en la Meseta oriental. Ed. La Ergastula. Madrid.

Bintliff, J. 2014: "Spatial analysis of past built environment: houses and society in the Aegean from the Early Iron Age till the impact of Rome". 
En E. Paliou, U. Lieberwirth y S. Polla (eds.) 2014: Spatial Analysis and Social Spaces: Interdisciplinary Approaches to the Interpretation of Prehistoric and Historic Built Environments, Topoi Berlin Studies of the Ancient World 18, De Gruyer, Berlin, pp. 263-274.

Blanton, R. E. 1994: Houses and Households: A Comparative Study. Plenum Press. New York.

Cañavate, V. 2008: "La aplicación de análisis arqueotectónicos en la arquitectura doméstica emiral del Tolmo de Minateda (Hellín, Albacete)". Lucentum XXVII, pp. 121-130.

Cañavate, V. 2013: "Aplicaciones metodológicas al estudio de la vivienda islámica”. En S. Gutiérrez e I. Grau (eds.): De la estructura doméstica al espacio social. Lecturas arqueológicas del uso social del espacio. Servicio de publicaciones de la Universidad de Alicante. Alicante, pp. 313-324.

Carandini, A. 2010: Le case del potere nell'antica Roma. Laterza. Roma.

Carvalho, R. y Penn, A. 2004: "Scaling and Universality in the micro-structure of Urban Space”. Physica A 332, pp. 539-547.

Cervera Vera, L. 1976: Sobre las ciudades ideales de Platón. Real Academia de San Fernando. Madrid.

Cervera Vera, L. 1987: "Los conceptos asimilados por Hipódamo de Mileto para su ciudad ideal". Boletín de la Real Academia de Bellas Artes de San Fernando 64, pp. 119-156.

Chatford Clark, D. L. 2007: "Viewing the liturgy: a space syntax study of changing visibility and accesibility in the development of the Bizantine church in Jordan”. World Archaeology 39 (1), pp. 84-104.

Coarelli, F. 2008: Rome and Environs: An Archaeological Guide.

Collins, W. N. 2005: "Centeredness as a cultural and grammatical theme in Maya-Nam". Unpublished PhD. Department of Linguistics. Ohio State University.

Craane. M.L. 2007: "Analysing Medieval Urban Space; a methodology". Internet Archaeology 21. http://dx.doi.org/10.11141/ia.21.5.

Dawson, P. C. 2002: "Space syntax analysis of central inuit snow houses". Journal of Anthropological Archaeology 21 (4), pp. 464-480.

Fairclough, G. 1992: "Meaningful constructions: Spatial and functional analysis of medieval buildings". Antiquity 66, pp. 348-366.

Feinman, G. 2012: "Comparative Frames for the Diachronic Analysis of Complex Societies: Next Steps”. En M. E. Smith (ed.): The Comparative Archaeology of Complex Societies. Cambridge University Press. Cambridge, pp. 21-43.

Fisher, K. D. 2009: "Placing social interaction: An integrative approach to analyzing past built environments". Journal of Anthropological Archaeology28, pp. 439-457.

Fisher, F. D. 2014: "Investigating monumental social space in Late Iron Age Cyprus: an integrative approach”. En E. Paliou, U. Lieberwirth y S. Polla (eds.) 2014: Spatial Analysis and Social Spaces: Interdisciplinary Approaches to the Interpretation of Prehistoric and Historic Built Environments, Topoi Berlin Studies of the Ancient World 18, De Gruyer, Berlin, pp. 167-202.

Funari, P. P. y Zarankin, A. 2003: "Social archaeology of housing from a Latin American perspective. A case study". Journal of Social Archaeology 3 , pp. 23-45.

George, M. 1995: "Review of Laurence, R. 1994: Roman Pompeii: Space and Society". Bryn Mawr Classical Review 95.10.19.

Grahame, M. 1997: "Public and Private in the Roman House: The Spatial Order of the Casa del Fauno". En R. Laurence y A. Wallace-Hadrill (eds.): Domestic Space in the Roman World: Pompeii and Beyond. Supplements of JRA, Portsmouth, RI, pp. 137-164

Grahame, M. 2000: Reading space: social interaction and identity in the houses of Roman Pompeii. BAR International Series 886, Archaeopress. Oxford.

Grau, I. 2013: "Unidad doméstica, linaje y comunidad: estructura social y su espacio en el mundo ibérico (ss. VI-I a.C.)". En S. Gutiérrez e I. Grau (eds.): De la estructura doméstica al espacio social. Lecturas arqueológicas del uso social del espacio. Servicio de publicaciones de la Universidad de Alicante. Alicante, pp. 57-76.

Gutiérrez, S. y Cánovas, P. 2009: “Construyendo el siglo VII: arquitecturas y sistemas constructivos en El Tolmo de Minateda”. En L. Caballero, P.
Mateos y M. A. Utrero (eds.): El siglo VII frente al siglo VII: arquitectura. Anejos de AEspA LI, CSIC. Madrid, pp. 91-132.

Hacigüzeller, P. 2008: "Modelling Human Circulation in the Minoan Palace at Malia”. En Herzog, I. (Eds.): Layers of Perception: proceedings of the 35th Conference of Computer Applications and Quantitative Methods in Archaeology (CAA), Berlin 2-6 April. Berlin, pp. 336-341.

Hacigüzeller, P. y Thaler, U. 2014: "Three tales of two cities? A comparative analysis of topological, visual and metric properties of archaeological space in Malia and Pylos". En Paliou, E.; Lieberwirth, U. y Polla, S. (Eds.) 2014: Spatial Analysis and Social Spaces: Interdisciplinary Approaches to the Interpretation of Prehistoric and Historic Built Environments, Topoi Berlin Studies of the Ancient World 18, De Gruyer, Berlin, pp. 203-262.

Hanson, J. 1998: Decoding Homes and Houses. Cambridge University Press. Cambridge.

Herzog, I. 2014: "Least-cost Paths - Some Methodological Issues". Internet Archaeology 36. http://dx.doi.org/10.11141/ia.36.5.

Hillier, B. 1985: "The nature of the Artificial: The Contingent and the Necessary in Spatial Form in Architecture". Geoforum 16 (2), pp. 163-178.

Hillier, B. 1989: "The Architecture of the Urban Object". Ekistics 334-335, pp. 5-21.

Hillier, B. 1996: "Cities as movement economies". Urban Design International 1 (1), pp. 41-60.

Hillier, B. 2002: “A Theory of the City as Object: How the social construction of Urban space is mediated by spatial laws". Urban Design International 7, pp. 181-203.

Hillier, B. 2014: "Spatial analysis and cultural information: the need for theory as well as method in space syntax analysis". En E. Paliou, U. Lieberwirth y S. Polla (eds.) 2014: Spatial Analysis and Social Spaces: Interdisciplinary Approaches to the Interpretation of Prehistoric and Historic Built Environments, Topoi Berlin Studies of the Ancient World 18, De Gruyer, Berlin, pp. 19-48.

Hillier, B. y Hanson, J. 1984: The Social Logic of Space. Cambridge UniversityPress. Cambridge.

Hillier, B. y Hanson, J. 1987: “Introduction: A Second Paradigm”. Architecture \& Behaviour 3 (3), pp. 197-199.

Hillier, B.; Hanson, J. y Graham, H. 1987: "Ideas are Things: An Applications of the Space Syntax Method to Discovering House Genotypes". Environment and Planning B: Planning and Design14, pp. 363-385.

Hillier, B.; Hanson, J. y Peponis, J. 1987: "Syntactic Analysis of Settlements". Architecture and Behaviour 3 (3), pp. 217-231.

Hillier, B. Penn, A.; Hanson, J.; Grajewski, T. y Xu, J. 1993: "Natural movement, or Configuration and Attraction in Urban Pedestrian Movement". Environment and Planning B: Planning and Design 20, pp. 29-66.

Hillier, B. y Vaugan, L. 2007: "The city as one thing". Progress in Planning 67 (3), pp. 205-230.

Hoepfner, W. y Schwandner, E.-L. 1994: Haus und Stadt im klassischen Griechenland. Wohnen in der klassischen Polis. Deutscher Kunstverlag. Munich.

Hudson, E. J. 2012: "Walking and Watching: New Approaches to Reconstructing Cultural Landscapes through Space Syntax Analysis". En D. A. White y S. L. Surface-Evans (eds.): Least cost analysis of social landscape: archaeological case studies. The University of Utah Press. Salt Lake City, pp. 83-99.

Isendahl, Ch. y Smith, M. E. 2013: "Sustainable agrarian urbanism: The lowdensity cities of the Mayas and Aztecs". Cities 31, pp. 132-143.

Jolivet, V.; Pavolini, C.; Tomei, M. A. y Volpe, R. (eds.) 2009: Suburbium II: Il suburbio di Roma dalla fine dell'Età monarchica alla nascita del sistema delle ville (V-II secolo a.C.). Collection de l'Ecole française de Rome 419. Roma.

Kaiser, A. 2000: The Urban Dialogue: An Analysis of the Use of Space in the Roman City of Empuries, Spain. BAR International Series 901, Archaeopress. Oxford.

Kaiser, A. 2011: Roman Urban Street Networks. Routledge Studies in Archaeology 2. Routledge. Londres.

Laurence, R. 2007: Roman Pompeii: Space and Society. $2^{\circ}$ Edition. Routledge. Londres. 
Laurence, R. y Newsome, D. (eds.) 2011: Rome, Ostia, Pompeii. Movement and Space. Oxford University Press. Oxford.

Leach, E. 1978: "Does Space Syntax Really “Constitute the Social?". En Green, D.; Haselgrove, C. y Spriggs, M. (eds.): Social Organization and Settlement: Contributions from Anthropology, Archaeology, and Geography. BAR International Series 47, Archaeopress. Oxford.

Lee, D.; Dias, E. y Scholten, H. J. (eds.) 2014: Geodesign by Integrative Design and Geospatial Sciences. Springer. Switzerland.

Letesson, Q. 2009: Du phénotype au génotype: analyse de la syntaxe spatiale en architecture minoenne (MMIIIB-MRIB). Aegis 2. Presses Universitaires de Louvain. Louvain-la-Nueve.

Letesson, Q. 2013: "Minoan Halls: A Syntactical Genealogy". AJA 117 (3): 303-351.

Letesson, Q. 2014a: "From Building to Architecture: The Rise of Configurational Thinking in Bronze Age Creta". En E. Paliou, U. Lieberwirth y S. Polla (eds.) 2014: Spatial Analysis and Social Spaces: Interdisciplinary Approaches to the Interpretation of Prehistoric and Historic Built Environments, Topoi Berlin Studies of the Ancient World 18, De Gruyer, Berlin, pp. 49-90.

Letesson, Q. 2014b: "Fire and the Holes: An Investigation of Low-Level Meanings in the Minoan Built Environment". Journal of Archaeological Method and Theory 22, pp 713-750.

Manzanilla, L. 1996: "Corporate groups and domestic activities at Teotihuacán”. Latin American Antiquity 7 (3), pp. 228-246.

Milnor, K. 2005: Gender, Domesticity, and the Age of Augustus: Inventing Private Life. Oxford University Press. Oxford.

Mol, E. 2012: Hidden Complexities of the Frankish Castle: Social Aspects of Space in the Configurational Architecture of Frankish Castles in the Holy Land, 1099-1291. Archaeological Studies Leiden University 25. Leiden.

Morton, S. G. 2007: Procession ritual at Naachtum, Guatemala during the Late Classic Period. Unpublished MA Thesis. Department of Archaeology. University of Calgary.

Morton, S. G.; Peuramaki-Brown, M. M.; Dawson, P. C. y Seibert; J. D. 2012: "Civic and household community relationships at Teotihuacán, México: a space syntax approach". Cambridge Archaeological Journal 22 (3), pp. 387-400.

Morton, S. G.; Peuramaki-Brown, M. M.; Dawson, P. C. y Seibert; J. D. 2014: "Peopling the past: Interpreting Models for Pedestrian Movement in Ancient Civic-ceremonia Centres". En S. Rau y E. Schönherr (eds.): Mapping Spatial Relations: Perceptions and Dynamics. Springer. Switzerland, pp. 25-44.

Neff, M. K. 2010: Modeling Space and Movement in a Prehispanic City: Cerro Jazmin, Oaxaca, Mexico. Unpublished Master Thesis, Department of Anthropology, Northern Arizona University, Flagstaff.

Nevadomsky, J.; Lawson, N. y Hazlett, K. 2014: “An Ethnographic and Space Syntax Analysis of Benin Kingdom Nobility Architecture". African Archaeological Review 31 (1), pp. 59-85.

Orhun, D., Hillier, B. y Hanson, J. 1996, "Socialising spatial types in traditional Turkish houses". Environment and Planning B: Planning and Design 23 (3), pp. 329-351.

Osman, K. M. y Suliman, M. 1994: “The Space Syntax Methodology: Fits and Misfits". Architecture and Behaviour 10 (2), pp. 189-204.

Osman, K. M. y Suliman, M. 1995:"SpaceSyntax: A Modified Algorithm for the Analysis of Non- Western Houses". Open House International 20 (4), pp. 46-53.

Paliou, E.; Lieberwirth, U. y Polla, S. (eds.) 2014: Spatial Analysis and Social Spaces: Interdisciplinary Approaches to the Interpretation of Prehistoric and Historic Built Environments, Topoi Berlin Studies of the Ancient World 18, De Gruyer, Berlin.

Penn, A. 2003: "Space syntax and Spatial Cognition: Or why the Axial Line?". Environment and Behavior 35 (1), pp. 30-65.

Pérez Rodríguez, V.; Anderson, K. C. y Neff, M. K. 2011: “The Cerro Jazmín Archaeological Project: investigating prehispanic urbanism and its environmental impact in the Mixteca Alta, Oaxaca, Mexico". Journal of Field Archaeology 36 (2), pp. 83-99.

Pesando, F. 1989: La Casa dei Greci. Longanesi. Milán.
Peuramaki-Brown, M. M. 2012: The Integration and Disintegration of Ancient Maya Urban Centers: Charting Households and Community at Buenavista del Cayo, Belize. Unpublished PhD. Department of Archaeology. University of Calgary.

Richards-Risseto, H. 2012: "Social Interaction at Maya Site of Copán, Honduras: A Least Cost Approach to Configurational Analysis". En D. A. White y S. L. Surface-Evans (eds.): Least cost analysis of social landscape: archaeological case studies. The University of Utah Press. Salt Lake City, pp. 109-127.

Seibert, J. D. 2006: Classic Maya Range Structures and Socio-political organization. Unpublished $\mathrm{PhD}$. Department of Archaeology. University of Calgary.

Sennett, R. 2004: "Las ciudades norteamericanas: planta ortogonal y ética protestante". Bifurcaciones 1. www.bifurcaciones.cl/001/reserva.htm

Shapiro, J. S. 2005: A Space Syntax Analysis of Arroyo Hondo Pueblo, New Mexico. Community Formation in the Northern Rio Grande. School of American Research Press. Santa Fe, NM.

Smith, M. E. 2003: "Can we read cosmology in Ancient Maya city plans? Comment on Ashmore and Sabloff'. Latin American Antiquity 14 (2): 221-228.

Smith, M. E. 2007: "Form and Meaning in the Earliest Cities: A New Approach to Ancient Urban Planning". Journal of Ancient Planning History $6(1)$, pp. 3-47.

Smith, M. E. 2011: "Empirical Urban Theory for Archaeologists". Journal of Archaeological Method and Theory 18, pp. 167-192.

Smith, M. E. y Peregrine, P. 2012: “Approaches to Comparative Analysis in Archaeology". En M. E. Smith (ed.): The Comparative Archaeology of Complex Societies. Cambridge University Press. Cambridge, pp. 4-20.

Steadman, S. R. 1996: "Recent research in the Archaeology of Architecture: Beyond the foundations". Journal of Archaeological Research 4 (1), pp. 51-93.

Stockett, M. K. 2005: “Approaching social practice through access analysis at Las Canoas, Honduras”. Latin American Antiquity 16, pp. 385-407.

Stöger, H. 2011: Rethinking Ostia: A Spatial Enquiry into the Urban Society of Rome's Imperial Port-Town, Archaeological Studies Leiden University 24. Leiden University Press, Leiden.

Stöger, H. 2014: "The spatial signature of an Insula neighbourhood of Roman Ostia", en E. Paliou, U. Lieberwirth y S. Polla (eds.), Spatial Analysis and Social Spaces: Interdisciplinary Approaches to the Interpretation of Prehistoric and Historic Built Environments, pp. 297-316. De Gruyter, Berlin and Boston.

Surface-Evans, S. L. y White, D. A. 2012: “An Introduction to the Least Cost Analysis of Social Landscapes”. En D. A. White y S. L. Surface-Evans (eds.): Least cost analysis of social landscape: archaeological case studies. The University of Utah Press. Salt Lake City, pp. 1-7.

Taaffe, E. J. y Gauthier, H. L. (eds.) 1973: Geography of Transportation. Prentice Hall. Englewood Cliffs. New Jersey.

Tanner, J. 1995: "Review of Laurence, R. 1994: Roman Pompeii: Space and Society and Wallace-Hadrill, A. 1994: Houses and Society in Pompeii and Herculanum". Antiquity 69, pp. 217-218.

Thaler, U. 2005: "Narrative and syntax: New perspectives on the Late Bronze Age Palace of Pylos, Greece". En A. Van Nes (ed.): Space Syntax. 5th International Symposium Conference. Delft, 13-17 June 2005. Amsterdam, pp. 323-339.

Turner, A.; Doxa, M.; O’Sullivan, D. y Penn, A. 2001: "From Isovist to Visibility Graphs: A methodology for the Analysis of Architectural space". Environment and Planning B: Planning and Design28 (1), pp. 101-121.

Ulrich, R. B. 1997: “"Review of Laurence, R. 1994: Roman Pompeii: Space and Society". Journal of Field Archaeology 24 (1), pp. 382-385.

Van Dyke, R. M. 1999: "Space syntax analysis at the Chacoan outlier of Guadalupe". American Antiquity 64 (3), pp. 461-473.

Van Nes, A. 2014a: "Space Syntax in Theory and Practice". En D. Lee, E. Dias y H. J. Scholten (eds.) 2014: Geodesign by Integrative Design and Geospatial Sciences. Springer. Switzerland, pp. 237-258.

Van Nes, A. 2014b: "Indicating street vitality in excavated towns. Spatial configurative analysis applied to Pompeii”. En Paliou, E.; Lieberwirth, 
U. y Polla, S. (Eds.) 2014: Spatial Analysis and Social Spaces: Interdisciplinary Approaches to the Interpretation of Prehistoric and Historic Built Environments, Topoi Berlin Studies of the Ancient World 18, De Gruyer, Berlin, pp. 277-295.

Vaquer, J. M. y Nielsen, A. E. 2011: “Cruz Vinto desde la superficie: alcances y limitaciones de la sintaxis espacial en un sitio del periodo de Desarrollos regionales tardío (ca. 1200-1450 d. C.) en el norte de Lípez, Potosí, Bolivia". Revista Española de Antropología Americana 41 (2), pp. 303-326.

Vaughan, L. Clark, DLC; Sahbaz, O; Haklay, M. 2005: "Space and exclusion: does urban morphology play a part in social deprivation?". Area 37 (4), pp. 402-412.

Wallace-Hadrill, A. 2002: "The Streets of Rome as Representation of Imperial Power". En De Blois, L. et al. (Ed.): The Presentation and Perception of Roman Imperial Power. J.C. Gieben. Amsterdam, pp. 189-206.

Westgate, R. 2007: "House and society in Classical and Hellenistic Crete: a case study in regional variation". AJA 111, pp. 423-457.

Weilguni, M. 2011: Streets, Spaces and Places. Three Pompeiian Movement Axes Analysed. Acta Universitatis Upsaliensis. Boreas, Uppsala
Studies in Ancient Mediterranean and Near Eastern Civilizations 33. Uppsala.

Zanker, P. 2000: "The City as Symbol: Rome and the Creation of an Urban Image". En E. Fentress (ed.): Romanization and the City: Creation, Transformation and Failures. JRA Suppl. Provindence. RI, pp. 25-41.

Zarankin, A. 1999: "Casa tomada: sistema, poder y vivienda doméstica". En A. Zarankin y F. Acuto (eds.): Sed Non Satiata: Teoría social en la Arqueología latinoamericano contemporánea. Ed. Del Tridente. Buenos Aires, pp. 239-272.

Zarankin, A. 2002: Paredes que domestican. Arqueología de la Arquitectura escolar capitalista: O caso de Buenos Aires. Centro de Historia da Arte e Arqueología. (IFCH-UNICAMP). Campinhas.

Zarankin, A. y Niro, C. 2006: "La materialización del sadismo. Arqueología de la Arquitectura de los centros clandestinos de detención de la dictadura militar argentina (1976-1983)". En A. Zarankin y P. P. Funari (eds.): $A r-$ queología de la represión y la resistencia en América latina, 1960-1980. Encuentro Grupo Editor. Córdoba, pp. 159-182. 